\title{
Characteristic Fluctuations around Stable Attractor Dynamics extracted from highly non-stationary Electroencephalographic Recordings.
}

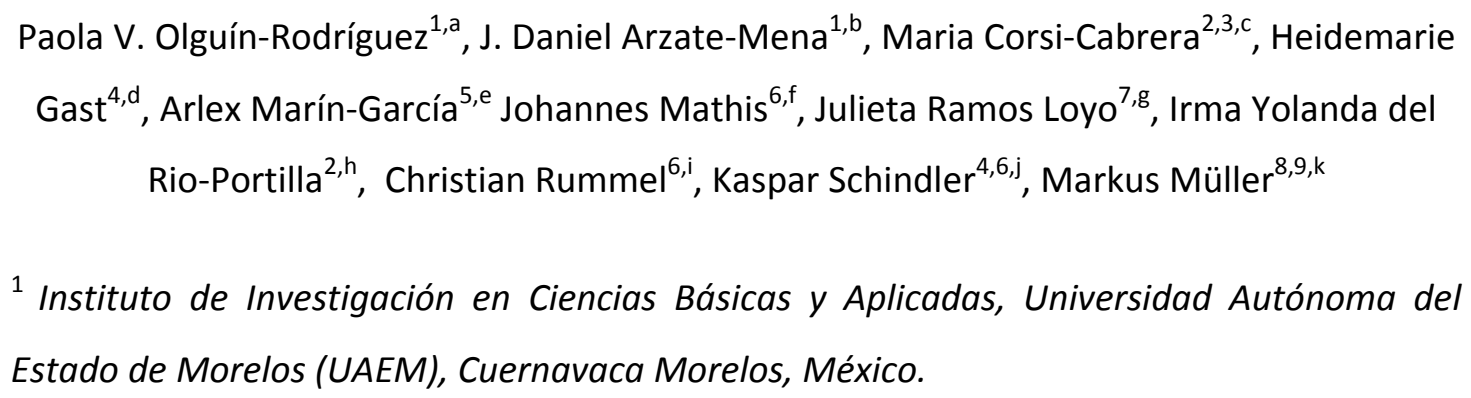
Estado de Morelos (UAEM), Cuernavaca Morelos, México.

${ }^{2}$ Facultad de Psicología, Universidad Nacional Autónoma de México (UNAM), Distrito Federal, México.

${ }^{3}$ Unidad de Neurodesarrollo, Instituto de Neurobiología, Universidad Nacional Autónoma de México (UNAM), Juriquilla, Querétaro, México

${ }^{4}$ Department of Neurology, Inselspital, Bern University Hospital, University Bern, Bern, Switzerland.

${ }^{5}$ Instituto de Ciencias Físicas (ICF), Universidad Nacional Autónoma de México (UNAM), Cuernavaca Morelos, México

${ }^{6}$ Support Center for Advanced Neuroimaging, University Institute for Diagnostic and Interventional Neuroradiology, Inselspital, Bern University Hospital, University Bern, Bern, Switzerland.

${ }^{7}$ Instituto de Neurociencias, Universidad de Guadalajara.

${ }^{8}$ Centro de Investigación en Ciencias, Universidad Autónoma del Estado de Morelos (UAEM), Cuernavaca Morelos, México.

${ }^{9}$ Centro Internacional de Ciencias A. C., Cuernavaca, Morelos, México 
ㅁane23.star@gmail.com, ${ }^{\mathrm{b}}$ jd.arzate@gmail.com, ${ }^{\mathrm{c}}$ corsi@unam.mx,

dheidemarie.gast@gmail.com, earlex.marin@gmail.com, fiohannes.mathis@belponline.ch, gjramos@cencar.udg.mx, hiyrp@unam.mx, ícrummel@web.de, jkaspar.schindler@gmail.com, ${ }^{\mathrm{k}}$ muellerm@uaem.mx

Corresponding author: Dr. Markus Müller

Address: Av. Universidad 1001 Col. Chamilpa C.P. 62209 Cuernavaca, Morelos, México Telephone: (777) 3297040 ext. 3273

e-mail:muellerm@uaem.mx

KEYWORDS:

Sleep, Epilepsy, EEG, Stationary Correlation Structure, Attractor Dynamics, Non-stationary Dynamics 
Abstract:

Since the discovery of electrical activity of the brain electroencephalographic recordings (EEG) constitute one of the most popular techniques of brain research. However, EEGsignals are highly non-stationary and one should expect that averages of the crosscorrelation coefficient, which may take positive and negative values with equal probability, (almost) vanish when estimated over long data segments. Instead, we found that the average zero-lag cross-correlation matrix estimated with a running window over the whole night of sleep EEGs, or of resting state during eyes-open and eyes-closed condition of healthy subjects shows a characteristic correlation pattern containing pronounced nonzero values. A similar correlation structure has already been encountered in scalp EEGsignals containing focal onset seizures. Therefore, we conclude that this structure is independent of the physiological state. Because of its pronounced similarity across subjects, we believe that it depicts a generic feature of the brain dynamics. Namely, we interpret this pattern as a manifestation of a dynamical ground state of the brain activity, necessary to preserve an efficient operational mode, or, expressed in terms of dynamical system theory, we interpret it as a "shadow" of the evolution on (or close to) an attractor in phase space. Non-stationary dynamical aspects of higher cerebral processes should manifest in deviations from this stable pattern. We confirm this hypothesis via a correlation analysis of EEG recordings of 10 healthy subjects during night sleep, 20 recordings of 9 epilepsy patients and 42 recordings of 21 healthy subjects in resting state during eyes-open and eyes-closed condition. In particular we show that the estimation of deviations from the stationary correlation structures provides a more significant differentiation of physiological states and more homogeneous results across subjects. 


\section{INTRODUCTION}

Zero-lag synchronization and desynchronization of neural activity is considered as a crucial communication mechanism of the central nervous system (Singer 1996, 1999; Engel and Singer, 2001; Varela et al., 2001; Fries, 2005). Such synchronization patterns may occur on various spatial scales, beginning from the integrated activity of several neuronal microcircuits up to the correlated dynamics of different cortical regions. The scalp EEG provides access to two spatial scales with excellent temporal resolution: the recording region of a single electrode and the largest spatial scale given by the coverage of the full set of scalp electrodes. While in the first case synchronization manifests in the amplitude and shape of EEG-oscillations of each individual EEG trace, the synchronization of the second scale, defining the functional network, is only accessible via the application of appropriate synchronization measures between multiple EEG traces.

On the other hand, cerebral electrical activity is highly non-stationary because of the permanent influence of alternating external stimuli and a continuous feedback from internal control mechanisms under ever changing conditions. Together with the high noise level of scalp recordings, this makes the quantitative analysis of EEGs a nontrivial task and large fluctuations of the results obtained for different recordings, in particular when measured for different subjects, is not an exception but rather common. For instance, even for clinically well defined epileptic seizures one observes an ample panorama of apparently contradictory results (Bartolomei et al., 2002, 2004; Wendling et al., 2003; Guye et al., 2006; Schindler et al., 2006, 2008; Müller et al., 2011). Contradictory reports of increasing or decreasing spatial interrelations during epileptic seizures may originate from different implantation schemes of intra-cranial recordings, different EEG-references, data preprocessing or the usage of different interrelation measures and underlines the large diversity and pronounced dynamical changes of the interrelation pattern during such events. In particular, there are pronounced qualitative changes of the functional network during epileptic seizures (see e.g. Schindler et al., 2008).

Resting state with open and closed eyes is markedly different in its power spectra (Barry et al., 2007) and BOLD dynamics (Zou et al., 2015). However, the difference of eyes open and 
eyes closed conditions during rest is not only given by changes of the e.g. Alpha-power (Kaur et al., 2017) but additionally, measures derived from graph theory indicate marked topological changes of the functional network (Tan et al., 2013). Also functional magnetic resonance imaging studies (fMRI) analyzing eyes-closed and eyes-open conditions reveal dynamical changes, expressed by the evolution of different correlation clusters (Allen et al., 2014, 2018; Damaraju et al., 2014).

Therefore, observations of temporal stable interrelation structures and uniform behavior across a group of subjects like in the case of the default mode network are evidently important. Most of those findings have been reported for fMRI-studies (Buckner et al., 2008; Fox et al., 2005; Greicius, 2008; Greicius et al., 2003; Honey et al., 2009; Jann et al., 2010; Nyberg et al., 1996; Raichle 2006; Raichle and Mintun, 2006; Raichle et al., 2001; Shulman et al.,1997), a technique with a limited ability to capture dynamical features due to its poor temporal resolution, and only few indications are published on the basis of EEG recordings.

In an early study (Corsi-Cabrera et al., 1997) the EEG have been recorded in a group of 9 young women in 11 sessions distributed over a period of one month in order to assess the within-subject and within-group stability of EEG correlations. The authors detected temporarily stable correlations of the EEG activity among electrodes, without significant differences between subjects. This result was confirmed by a second study (Corsi-Cabrera et al., 2007), where correlations have been estimated for a group of 6 young females over a period of 9 months. Within-subject reliability was very high $(r>0.89)$ and the results were homogeneous across the subject-group.

In He et al. (2008) a stable correlation pattern of slow cortical potentials as accessed by electrocorticography across wakefulness, slow wave and Rapid Eye Movement (REM) sleep has been shown in 5 epilepsy patients. Surprisingly, the correlation structure has been found to be similar to that of spontaneous fluctuations of blood oxygenation level dependent signals (BOLD), which suggests a direct link between measurements of blood oxygen consumption and electrical brain activity. Also Kramer et al. (2011) reported that stable network templates emerge after as little as 100 seconds. The authors claim 
topological stability, while several graph theoretical measures show dependency on physiological states and frequency bands.

In Müller et al. (2014), stable correlation structures have been observed in 20 scalp EEG recordings of 9 patients containing focal onset seizures. The authors show explicitly that this observation is not due to volume conduction, but reflect genuine interrelations stable in time. This skeleton of large scale interrelations, produced by permanent oscillatory neural background activity, assures simultaneously an efficient coordination of local functional networks as well as large scale integration and provides an adequate framework capable to coordinate bottom up as well as top down processes.

While the above mentioned early results have been obtained under comparable physiological conditions, the recent results have been found in epileptic brains. It is therefore impossible to determine if the stable interrelation pattern is a generic feature of the human brain, just an intrinsic expression of the pathology or the consequence of pharmacological treatment. Therefore, the question of the existence of a stationary pattern in non-pathological brains under normal but physiologically varying conditions persists.

Like epileptic seizures also during wakefulness, during sleep and transitions between both states constitute pronounced variations of brain function with profound electrophysiological changes and, hence, present an ideal testing ground for probing such hypothesis. For instance, REM-sleep is considered as a state of brain activation where EEGsignals show low voltage fast activity including Beta and Gamma oscillations (Rechtschaffen and Kales, 1968; LLinás and Ribary, 1993; Achermann 2016), whereas NonREM sleep (NREM) is characterized by high voltage, Delta and Theta oscillations containing special signatures like sleep spindles and K-complexes (Rechtschaffen and Kales, 1968; Steriade, 1997; Steriade and McCarley, 1990). In addition to changes of the power of ongoing electrical activity, the temporal coupling among brain regions is modified during sleep stages relative to wakefulness in a state-dependent manner (Corsi-Cabrera et al., 1987; Nielsen et al., 1990; LLinás and Ribary,1993; Guevara et al., 1995; Kaminski et al.,1997; Achermann and Borbely, 1998; Cantero et al 2000; Pérez-Garci et al., 2001; De 
Gennaro et al, 2001; Corsi-Cabrera et al., 2003, Duckrow and Zaveri, 2005; Voss et al., 2009). Additionally, we consider resting state EEG-recordings of healthy subjects while having eyes open and closed, two conditions with remarkable changes of the spectral contents of the electrical brain activity (Allen et al. 2018), i.e. with qualitatively different dynamical features. Hence, due to the fact that sleep recordings as well as the comparison of the eyes-closed and eyes-open state show pronounced changes of the morphology of EEG-signals, their spectral contents and the interrelation pattern of different brain regions makes them an appropriate testing ground for probing the existence of a stationary correlation structures. However, if there is a stationary pattern of functional interrelations among cortical areas in non-pathological conditions, it should be observable independently of the physiological state of a subject, while non-stationary dynamical aspects of interrelations should occur as deviations from the stationary pattern.

The first objective of this study is to investigate if a stationary pattern of functional connectivity exists under non-pathological conditions. This has been done by a quantitative comparison of average correlation matrices taken separately for different sleep stages and eyes-open/eyes-closed condition. Additionally, we performed a quantitative comparison with the results presented in (Müller et al. 2014) in order to provide further evidence for the independence of the observed pattern from the physiological brain state, even though derived from signals with severe pathological signatures.

Secondly, we evaluated in which manner the distance to the hypothesized stationary pattern may serve as an improved quantifier of dynamical changes in comparison to an established scheme of analysis.

We hypothesize that (1) if a stable pattern of functional connectivity exists, it will be present independently of the physiological state and shows a high similarity to those interrelation structures encountered in epilepsy patients; and (2) that transient dynamical features of the brain activity manifest themselves as deviations from the stable pattern. 


\section{Material and Methods}

\section{Experimental Design:}

In total we consider 72 recordings of 41 subjects, which are divided in 20 recordings of 9 epilepsy patients, 10 sleep recordings of 10 healthy subjects and 42 recordings of 21 healthy subjects in resting state during eyes-open and eyes-closed condition. Data have been recorded in four different labs, using different EEG-equipment and data preprocessing routines.

\section{Sleep Data}

10 right handed neurologically healthy subjects recorded in the Sleep Laboratory of the Faculty of Psychology of the National Autonomous University of Mexico participated in this study, after giving written informed consent. Table 1 provides information about the participants and the percentage they stayed in each sleep stage.

Before the study all subjects had a structured clinical interview and kept a 15-day sleep log. Only those with regular sleeping habits and no symptoms of sleep disorders, history of medical, psychiatric or neurological disorders, drug or medication were included. The protocol was approved by the Ethical Committee of the Faculty of Medicine of the National Autonomous University of Mexico and followed the ethical standards of the Declaration of Helsinki (1964).

All subjects slept two nights at the laboratory, the first for adaptation to recording procedures and the second for EEG analysis. Standard Polysomnography (PSG) and included a standard scalp EEG were recorded at Fp1, Fp2, F3, F4, F7, F8, C3, C4, T3, T4, T5, T6, P3, P4, O1, O2, Fz, Cz and Pz of the 10-20 International System (Lesser, 1986) referenced to A1 with a Grass 8-20 polygraph with filters set at 0.1 and $70 \mathrm{~Hz}$ for EEG, at 10 and 70 for EMG and 0.3 and $70 \mathrm{~Hz}$ for EOG. All night PSG data were digitized and stored with $1024 \mathrm{~Hz}$ sampling rate and using a 12-bit A-D converter of the GRASS-GAMMA acquisition program. 
Wakefulness and Sleep stages were identified by standard procedures using 30 -second epochs (Rechtschaffen and Kales, 1968). Percentages were calculated over the total recording time of the whole night. Sleep stage percentages as shown in Table 1 correspond in general terms with the values expected for young adults (Williams et al., 1974).

\section{Epilepsy patients}

EEG data were recorded from nine patients (five men, four women, age range 21-45 years) suffering from pharmacoresistant temporal lobe epilepsy. Subjects have been under presurgical evaluation at the Department of Neurology of the Inselspital of the University of Bern. The ethics committee of the Kanton of Bern approved this retrospective study. Further, all patients gave written informed consent that their EEG data might be used for research and teaching purposes.

For the EEG recordings standard 10-20 montage positions (Lesser, 1986) were used. After passing an anti-aliasing filter with a cutoff frequency of $70 \mathrm{~Hz}$ and an attenuation of 24dB/oct, the EEG signals were sampled at $200 \mathrm{~Hz}$ (seizure 1-12) and $256 \mathrm{~Hz}$ (seizure 1320) using the earlobe reference. $A / D$ conversion had a resolution of 16 bit. EEG seizure onset and seizure offset were visually determined by an experienced electroencephalographer (K.S.) in bipolar montage. Table 2 provides information about patients and seizure durations.

Further information about the EEG recordings from epilepsy patients can be found in (Müller 2014).

\section{Healthy subjects in resting state during eyes-open and eyes-closed condition}

The EEG recordings during resting state were provided from two different laboratories and contain in total 21 subjects (see Table 3). In all cases subjects were in resting state for two minutes with closed eyes and open eyes, respectively. When eyes open they were instructed to look at a mark in the center of a computer screen. All the subjects gave written informed consent. 
11 elderly healthy subjects (five men, six women, age range 67-94 years) has been measured in the Laboratory of the Clinical Neurophysiology of the Institute National of Medical Science and Nutrition Salvador Zubirán INCMNSZ. By using the 10-20 International System (Lesser, 1986) standard scalp EEG at Fp1, Fp2, F3, F4, F7, F8, C3, C4, T3, T4, T5, T6, $\mathrm{P} 3, \mathrm{P} 4, \mathrm{O} 1, \mathrm{O} 2, \mathrm{Fz}, \mathrm{Cz}$ and $\mathrm{Pz}$ referenced to earlobe with filter set at 0.1-70 Hz and the signal were sampled at $200 \mathrm{~Hz}$.

The other 20 EEGs data in resting state (closed eyes and open eyes) of healthy subjects (seven women, three men, age range $23-38$ years) were recorded in the Laboratory of Psychophysiology of Cognitive and Emotional Processes of the Institute of Neuroscience of the University of Guadalajara, Mexico by using an Electro-Cap with the same 19 electrodes positions of the 10-20 International Systems as mentioned above. The signals were referenced to earlobe with filter set at $0.1-70 \mathrm{~Hz}$ and sampled at $200 \mathrm{~Hz}$ and using Neuronic acquisition program.

EEG data of all recordings (sleep data, resting state as well as recordings from epilepsy patients) were transformed to median reference (Müller et al. 2011, 2014). All EEG signals were filtered to obtain a broad band ranging between 0.5 and $25 \mathrm{~Hz}$ in order to diminish the influence of muscle artifacts by using a fourth order Butterworth filter. Fp1, Fp2, 01 and 02 were excluded from the analysis because usually these electrodes are most contaminated by blink and eye movements, and muscular artifacts respectively.

\section{Statistical Analysis:}

Nowadays there exists a broad palette of different interrelation measures in order to construct the functional brain network (Boccaletti et al. 2006), usually divided in linear and nonlinear estimators (Galka 2000; Kantz and Schreiber 2004; Pereda et al., 2005). Due to the fact that single neurons, the elementary building blocks of the brain, show pronounced nonlinear dynamical properties (Keener and Sneyd,1998) one might expect that nonlinear estimators are better suited for the extraction of relevant features from empirical time series. However, for interrelation properties it was proven that even for the favorable situation of low-dimensional stationary nonlinear systems the performance of linear cross- 
correlations is highly competitive with several commonly used nonlinear measures stemming from different areas like information theory, phase-space reconstruction or synchronization measures (Kreuz et al., 2007, Mormann et al., 2005). Also in Ansari-Asl et al. (2006) it was demonstrated that numerically robust but simple linear measures may perform better than sophisticated algorithms, which aim to extract linear as well as nonlinear interrelations. Based on these findings we mainly focus in the present study on the estimation of zero-lag cross-correlations. Maximum lag correlations are computed additionally to provide evidence that the observed effects are not trivial artifacts of volume conduction.

In all practical circumstances cross-correlations are estimated over finite data segments, whose length is adjusted in order to balance between stationarity requirements and statistical accuracy. For a multivariate data set ofMEEG channels the zero-lag crosscorrelation matrix is estimated by:

$$
C_{i j}=\frac{1}{T} \sum_{k=1}^{T} X_{i}\left(t_{k}\right) X_{j}\left(t_{k}\right)
$$

HereTdenotes the number of samples of the data segment andi, $j=1, \ldots$, Mare electrode numbers. In formula (1) the data is normalized to zero mean and unit variance, such that the correlation coefficient takes values between \pm 1 . All diagonal elements are equal to one because each signal is perfectly correlated with itself. Furthermore, the matrix is real symmetric and, being a quadratic form, positive semi-definite. By using a running window approach the correlation matrix may be estimated in a time dependent fashion.

Equation (1) can be understood as an average over the product of real values. If the two sets $X_{i}(k), X_{j}(k)$ are independent random variables, the correlation coefficient is precisely zero in theory. However, as the sum in Eq. (1) is taken over a finite range, numerical estimates are distributed symmetrically around zero (Mueller 2011). Furthermore, the shorter the data segments, the larger the probability that non-zero estimates take values notably different from zero. In fact the situation is even worse. The magnitude of non-zero estimates depends not only on the length of the data window but also on the spectral content of the data, which may change drastically during the time course of an EEG- 
recording (Müller 2011). The larger the contribution of slow frequency components of a signal, the larger is the amount of random correlations (Rummel et al., 2010). This phenomenon, caused by the fact that cross-correlations are estimated over finite data segments (and in many practical applications over quite short segments in order to improve time resolution), is called "random correlation" (Plerou et al., 1999; Laloux et al. 1999).

In Marín-García et al. (2013) a method is proposed, which aims to obtain reliable estimates for genuine correlations with a well defined significance level, based on the use of appropriate surrogate data. It turned out that the so called Significant Average Correlation Matrix (SAC) performs best in terms accuracy of the results, the sensitivity to detect correlations and the robustness against noise, in comparison to several other proposals. The matrix elements of the SAC-matrix are defined as:

$$
S A C_{i j}(t)=\frac{S_{i j}}{N_{T}} \sum_{k=1}^{N_{T}} C_{i j}^{k}(t)
$$

The sum is just the average over matrix elements of the cross-correlation matrix estimated over $N_{T}$ data windows, each of lengthT, while $S_{i j}$ represents the result of a non-parametric significance test. To this end a set of Shift-Surrogates (Nettoff et al. 2002) is created from the original data segment. Then each segment is divided in $N_{T}$ data windows, and, like in the case of the original data, for each window the zero-lag cross-correlation matrix is estimated. Thereafter, we applied for each matrix element separately the Mann-WhitneyWilcoxon rank test in order to estimate the probability that correlation coefficients derived from the original data and the surrogates belong to distributions with the same median values. Including a Bonferroni correction for multiple testing at a $1 \%$ significance level, $S_{i j}$ was set to one if $p<\frac{0.01 * 2}{[M(M-1)]}$. Otherwise $S A C_{i j}(t)$ was set to zero.

In order to probe the hypothesis, of the existence of a pronounced correlation pattern that is stable in time like in Müller et al. (2014) and, universal in the sense of a high similarity across subjects, we studied similarity between average genuine correlation matrices. In the case of the sleep data, we used 30 seconds segments that coincided with the so called sleep epochs used by experienced electroencephalographers (M.C. and I.Y.R) for sleep 
scoring. These were divided into $T=3$ snon-overlaping data windows in order evaluate formula (2). We estimate the average matrices separately for each sleep stage. In case of recordings from epilepsy patients and resting state EEG during eyes-open and eyes-closed condition we used segments of length 10 seconds, which are divided in $N_{T}=10$ nonoverlapping data windows of length $T=1 s$. Due to the filtering, the short windows contained 3 and $1 / 2$ cycles of the slowest retained waves in all cases.

Note, the large length of a segment is due to the fact that in the present contribution exclusively broad band signals are considered. For the analysis of e.g. Alpha-activity much shorter segments of about $1-2$ seconds are already appropriate.

In order to quantify the statistical similarity of two average correlation matrices $\left\langle S A C_{n}\right\rangle$ and $\left\langle S A C_{m}\right\rangle$ we first ordered their non-diagonal elements in one dimensional vectors $V_{n}$ and $V_{m}$ by the "vech" operation. These objects are called matrix vectors in the sequel. After normalizing these vectors to zero mean and unit variance we estimated the pairwise correlation between them via formula (1). Furthermore, we applied the nonparametric Mann-Whitney-Wilcoxon-rank test, which estimates the probability that the median off-diagonal matrix element of the two SAC matrices are different (a nonparametric test whether the median of the correlation coefficients is different). This test has the advantage that no assumption about the underlying probability distributions of the samples is done, but the discrimination power is in general reduced when compared with parametric test statistics. Thus, estimated $p$-values in this paper can be considered as upper limits.

Note, the Pearson-coefficient estimated for different SAC-matrices quantify structural similarity between these matrices but due to normalization it is insensitive for differences in the magnitude by which a certain correlation pattern is expressed. In order to test statistical equivalence also for the overall strength of the stationary pattern we applied additionally, the Mann-Whitney-Wilcoxon-rank test.

If no significant differences between averaged SAC-matrices estimated separately for different physiological stages can be found, we concluded that the average over the whole 
14

recordings is justified. The result of this overall average is termed "stationary pattern" in the sequel and will be denoted by $\langle S A C\rangle$. The stationary pattern of the peri-ictal recordings of epilepsy patients has already been evaluated in a similar fashion (Müller et al. 2014).

In order to probe stability in time and further to test the hypothesis that dynamical features are imprinted in deviations from $\langle S A C\rangle$ we focus in the second part of this work on the time evolution of genuine cross-correlations, and likewise differences to the stationary correlation structure, by employing a running window approach.

We estimated the SAC-matrix using a running window approach along the whole recordings, with a step-width of 30 seconds for sleep data and 10 seconds epilepsy data and recordings of eyes-open and eyes-closed condition during rest. For each window we then computed the correlation between the SAC-matrix and the stationary pattern $\langle S A C\rangle$.In order to summarize the results concerning the temporal stability of the stationary pattern of all subjects we provided medians and the $95 \%$ confidence interval of the Pearson correlations estimated separately for each sleep stage, eyes-open and eyesclosed condition or respectively, separately for the pre-ictal, ictal and post-ictal interval. Small confidence intervals are then indicators for a high temporal stability of $\langle S A C\rangle$ across all subjects.

Finally, to probe our second hypothesis we determined in which manner deviations from the stationary pattern were capable to distinguish different physiological states and compared the results to a classical strategy, namely cross-correlation values itself. For this purpose, we calculated the average of the absolute value of the non-diagonal elements of the SAC-matrices

$$
\left\langle\left|S A C^{l}\right|\right\rangle=\frac{2}{M(M-1)} \sum_{i>j}^{M}\left|S A C_{i j}^{l}\right|
$$

obtained for a particular sleep stage, for the eyes-open and eyes closed condition or respectively the different phases of the peri-ictal transition, across all subjects. Thereafter 
we computed from this set of real numbers the median and the $95 \%$ confidence interval separately for each physiological state.

Finally we also considered deviations from the average matrix (2)

$$
D_{i j}(t)=S A C_{i j}(t)-\left\langle S A C_{i j}\right\rangle
$$

Note, in order to evaluate characteristic deviations of a particular physiological stage, this difference matrix can be used in (3) instead of $S A C_{i j}$.

\section{Model Calculations:}

In order to substantiate the interpretation of the observed phenomena, which we provde in the discussion section, we performed additionally some model calculations of explicit non-stationary dynamical systems. In a first step we estimated the correlation matrix of two strongly coupled Rössler systems, were each of the variables of the six dimensional phase space were erratically perturbed by independent Gaussian white noise. Because the random perturbations have been frequently applied (every 250 time steps) the system is almost permanently located on a transient around the attractor and is thus non-stationary by definition. Then we estimated the average of the six-dimensional correlation matrix and compared the outcome with the equivalent non-perturbed system.

In the second case we deformed the attractor (actually we consider a mixed system) showing that in this case the correlation matrix strongly depend on such deformations. Here time series are derived as a mixture of the strongly coupled Rössler system and a system of two anti-correlated Lorenz oscillators. The mixture has been varied gradually along the time course such that at the first time step the recording consisted of the pure Rössler systems and at the last time step the anti-correlated Lorenz systems remain. In this way we permanently deform the invariant set of the whole system.

A detailed description of the differential equations, chosen parameters as well as the numerical results can be found in the supplementary material. 
Results:

To illustrate certain details of our analysis we present results of one healthy subject during sleep (subject 9 of Table 1) before we provide summary figures merging the results of all recordings. We start with the search of a pronounced temporarily stable correlation structure.

\section{Existence of a Pronounced Stationary Correlation Pattern:}

In a first step we searched for stationary correlation pattern. Based on the findings published in Müller et al. (2014), such pattern is supposed to be independent of the physiological state, viz. a particular sleep stage. In Figure 1 we show mean correlation matrices from the recording of subject 9, where averages have been taken over all 30 second segments of the whole night, but separately for each sleep stage. A similar figure of a young and an old adult with eyes open and eyes closed is presented in the supplementary material (Fig.S1).

In close similarity with findings derived from scalp EEG recordings of epilepsy patients (Müller et al. 2014) we here observe a pronounced correlation structure in all sleep stages (as well as for eyes-open and eyes-closed condition), in spite of the marked electrophysiological and neurophysiological differences between REM, non-REM and the awake state. Like in (Müller et al. 2014), positive correlations are more prominent for connections within the same hemisphere, while diagonal contra-lateral electrode pairs tend to be anti-correlated. Considering furthermore the high noise level of scalp EEGs and the non-stationarity of the brain activity the appearance of high magnitude average crosscorrelations is a counter intuitive result.

The intriguing similarity of the pronounced correlation patterns shown in Figure 1 leads to the question whether an average over the whole recording without distinguishing sleep stages is justified. In order to substantiate such procedure we estimated for all subjects the correlation between all pairs of the average correlation matrices obtained separately for the sleep stages. We found that average SAC-matrices of different sleep stages are indeed highly correlated with Pearson coefficients above 0.9. Furthermore, we compared 
quantitatively the equivalence of the median off diagonal matrix elements by using the Mann-Whitney-Wilcoxon-rank test, without finding significant differences. In consequence, we found that in spite of drastic physiological differences, marked morphological changes of the EEG-recordings between sleep stages as well as alternations of the functional network, we observed a similar pronounced average correlation pattern. The same is true for the comparison of the eyes-open and eyes-closed condition. Pearson coefficients encountered surprisingly high estimates. Consequently, we conclude according to these findings that the average over the whole recording, termed as "stationary pattern" in the sequel, is justified. Corresponding results and a detailed discussion can be found in the supplementary material (see Figure S2 and S3 in the Supplementary Material).

\section{Stability over time and temporal fluctuations:}

In the next step we study the temporal stability of the correlation coefficients in order to further probe for the stationarity of the average correlation pattern. For this purpose, we estimated the correlation between the stationary pattern and SAC-matrices estimated with a running window approach and visualized the time course of all 105 matrix elements of the SAC-matrix. Results for subject 9 are provided in Figure 2.

In Figure 2 we observe that the correlation between the SAC-matrix and the stationary pattern is almost always above 0.85 , which constitutes an extremely high value. Only at the beginning of the recording one notices a gradual increase of correlations up to values of about 0.95 . After this initial phase the correlation appears to be quite stable and even outliers (for example during short waking stages between minute 250 and 300) never drop below 0.6 , which still implies a pronounced similarity. Furthermore, such minimal values are encountered only for short moments and appear as sharp spikes in Figure 2. Beside of such short episodes the correlation of the time depended SAC matrix with the stationary pattern is considerably high and fluctuates around 0.9.

The visual inspection of the time course of the SAC-matrix elements (upper panel of Figure 2 ) confirms these results. Despite pronounced changes of the spectral composition as well as significant topological alternations of the functional network during sleep cycles (Corsi- 
Cabrera et al., 1987; Nielsen et al., 1990; LLinás and Ribary,1993; Guevara et al., 1995; Kaminski et al.,1997; Achermann and Borbely, 1998; Cantero et al 2000; Pérez-Garci et al., 2001; De Gennaro et al, 2001; Corsi-Cabrera et al., 2003, Duckrow and Zaveri, 2005; Voss et al., 2009, Gast et al. 2014) the majority of the matrix elements never change its sign and fluctuations around some non-zero constant value seem negligible. Matrix elements with a low average magnitude show noteworthy fluctuations, while the overall impression of Figure 2 resembles a remarkably stable correlation pattern. Even transitions between sleep stages are hardly visible.

Note, this observation does not imply a lack of interesting dynamical features imprinted in the EEG-signals and we do not challenge the highly non-stationary character of such recordings. On the contrary, we believe, that the extraction of such non-stationary features provide important and novel progresses for the understanding of the brain dynamics. On this ground the observation of a stable scaffold of functional connectivity is even more surprising.

The results obtained for all subjects (Figure 3) corroborate these observations. For all sleep stages the medians are about 0.85 and fluctuations are remarkably small. Only for the waking state (and partly for the transitional stage 1 ) one observes a considerable amount of fluctuations. However, the median of the correlation coefficient during waking state is about 0.87 and the lower value of the $95 \%$ confidence interval of the cross-correlations is still larger than 0.5. Furthermore one observes that the fluctuations during sleep stages are not generated by a large discrepancy between subjects. Median values estimated for each subjects separately are almost always above 0.9. Only a few subjects and only during sleep stages 3 and 4 encounter values between 0.85 and 0.9 and also the comparably large fluctuations during the awake state can not be explained by variations across subjects, given that, beside of the exception of two subjects, all medians are clearly above 0.85 . However, $90 \%$ confidence intervals are considerably larger for the wake state than for all sleep stages.

Based on these results one may conclude that there exists a kind of rigid skeleton of oscillatory interrelations between brain regions covering the whole scalp. This spatially 
extended but stable structure could be advantageous for an efficient communication between different, probably distant brain sites. On one hand it might coordinate global dynamical features of widely interconnected neuronal populations and additionally it may admit that local deformations of this structure quickly propagate in order to cover larger spatial scales. Hence, such a skeleton could balance between segregation of local activity and global information integration (Singer 1999; Tononi et al. 1998, Tononi 2004, 2010). Consequently, this picture suggests that transient dynamical features should manifest themselves via deviations from this structure. Accordingly, one should expect that the waking state, with a major variety of different kind of activity, is characterized by larger fluctuations around the stationary pattern as shown in Figure 3.

\section{Subject specific or generic feature}

It remains the question whether the pronounced stationary correlation pattern represents individual signatures of the brain dynamics of a single person, like a stationary dynamical fingerprint of neural activity, or, whether it is a generic phenomenon, which reflects general principles of brain functioning. In order to answer this question we compare in Figure 4 the stationary pattern obtained for each EEG recording, viz. Sleep and resting state EEGs as well as the epilepsy recordings.

In (Müller et al. 2014) it was already found that mean correlation matrices, estimated over periods of several minutes, show also a marked correlation pattern, which, as in the present case, seem to be independent of the physiological state of the subject. Namely, average matrices derived from the pre-seizure, post-seizure or the seizure period show a remarkable similarity (Pearson correlations are always higher than 0.6, Fig. 3 of (Müller et al. 2014) and also peri-ictal averages of different subjects are surprisingly similar. Pearson coefficients are larger than 0.5, (see Fig. 4 of (Müller et al. 2014)). Smallest values are obtained exclusively for the comparison with recording 20 of the epilepsy patients, which already has been identified as an outlier (Müller et al. 2014). Furthermore, by visual inspection one gains the impression that the stationary pattern obtained from this pathological activity shares many features with the structures observed for the sleep data. 
The Pearson coefficient quantifies topological similarity between the average correlation pattern, which turns out to be considerably high for all cases (Figure 4.A). Only for the pairwise comparisons with the above mentioned recording 20 of the epilepsy patients and one recording of the young adults during resting state (OE y CE-condition) the correlation values drop slightly below 0.5 , while in general Pearson coefficients are considerably above 0.6. Furthermore, a certain structure of the matrix shown in Fig. 4.A can be identified. First one can observe that the sleep EEG segregate in two cluster (subject 1-3 and 4-10), where the stationary pattern are extremely similar within these clusters, given that the Pearson correlations take values about 0.9 , while inter-cluster correlations are in general smaller. Furthermore, one identifies a high similarity between the second cluster and almost all remaining recordings, independently whether the pathological case of epilepsy or healthy subjects during rest are considered. The peri-ictal average of the seizure EEGs, on the other hand, correlate stronger with the resting state EEGs of older subjects than those obtained for young adults, independently where eyes are open or closed during rest. But most importantly one observes non-ambiguously that also resting state EEGs fall into two similarity clusters, which separate young and old adults. While almost no difference between EO and EC-condition can be noticed for both groups, Pearson coefficients between these groups are systematically lower, which may indicate that although the stationary pattern seemingly does not depend on the physiological state, it may evolve with the age of the subjects.

While the Pearson coefficient is sensitive for the topological differences of the correlation pattern, the application of the Mann-Whitney-Wilcoxon-rank test probes similarity of the correlation strength. For comparison and, hence, as a kind of null-hypothesis the cumulative distribution of $p$-values for the comparison of white noise samples is drawn. Note, the cumulative distribution of the white noise data indicate the probability that $\mathrm{p}$ values occur solely by chance. Hence, a necessary condition for significance is that the smallest $p$-values should lie above the gray straight line.

In Fig. 4.B we compare average correlation matrices obtained for the same condition as e.g. all 45 pairwise comparisons of the sleep EEGs. As a result, within the same condition no significant differences can be found. However, when EEGs obtained for different 
conditions are compared, the sleep EEGs stand out (Fig. 4.C). Independently whether they are compared with epileptic seizures, or with the eyes closed and eyes open condition, the cumulative distributions are above the null hypothesis of white noise samples. However, for small p-values all three curves drop below the white noise model such that only a few comparisons encounter sufficiently small p-values (see inset of Fig. 4.C). Thus, although the three curves for the comparison with sleep-EEGs are notable above the null hypothesis for a large range of $p$-values, no significant differences between physiological conditions can be found. In addition, the results shown in Figure 4 involve multiple testing and require a Bonferroni correction, which lessens the critical p-value by a factor of 200 for the comparison of the sleep and epilepsy data. Thereby none of the obtained p-values mark significant differences, a result which is corroborated by the estimation of Pearson coefficients (Fig 4.A).

In summary, one observes that the similarity between the stationary pattern of the sleep or resting state EEGs and the peri-ictal averages of epileptic seizures is high. Furthermore, one notice that the resting state EEGs fall into two correlation clusters, which do not distinguish between eyes open and eyes closed condition but separate subjects with a notable age difference. Consequently, the high similarity between the average correlation matrices and the apparent statistical equivalence of the set of matrices lead us to the conclusion that the observed pattern is a generic feature and may reflect universal principles of the brain dynamics, which may evolve with the age of the subjects, although we do not provide sufficient quantitative support for this last suspect.

\section{Discrimination between different physiological states}

In order to test the hypothesis that non-stationary dynamical features are imprinted in the deviations from the stationary pattern, we compared the different sleep stages, resting state for open and closed eyes as well as the pre-ictal, ictal and post-ictal phase by estimating the Pearson coefficient of pairs of the difference matrices (4), averaged separately for different physiological states for each of the subjects. These results should be compared with those obtained for the SAC-matrix shown in Figure S.2 and S.3 of the supplementary material as well as those presented in Fig. 3 in (Müller et al. 2014). 
The Pearson coefficients for the comparison of the average difference matrix obtained for sleep and wakefulness are shown in Fig 5. In contrast to Figure S.1 and S.2, the color scales of Figure 5 cover now a much more extended range and thus the differences matrices are distributed over a much wider range, which implies that deviations from the stationary pattern constitute an improved discrimination of sleep stages. Results shown in Figure 5.A and $B$ fit to neurophysiological findings about sleep-wake cycles. NREM sleep stages 3 and 4, and to a lesser degree Stage 2, are highly correlated, which is consistent with the fact that they belong to the same Thalamo-cortical oscillatory mode (Steriade and McCarley 1990). Furthermore, one observes that pairs of average difference matrices may be anticorrelated, which indicates that deviations from the average correlation pattern are toward opposite directions in matrix space. For example, anti-correlations are observed for the comparison between the three NREM sleep stages and activated states W and REM as expected from the different physiology of REM, W and NREM sleep (Steriade and McCarley, 1990). Also the mean difference matrix obtained for stage 1 is anti-correlated to those of stages 3 and 4 . If dynamical aspects are imprinted in the deviations from the stationary pattern, the dynamics of anti-correlated sleep stages are qualitatively different. On the other hand, REM sleep and Stage 1, as well as REM sleep and W share rather similar features, which is consistent with the activated electro-physiologic pattern of REM sleep, which hinders the differentiation of $W$ and Stage 1 sleep. Until the rapid eye movements and the muscle atony allows to recognize them as different brain states with its own physiological characteristics (Steriade and McCarley, 1990).

It is conspicuous that stage 2 sleep is identified as qualitatively different from stages 3 and 4 in some subjects. The same is true for the comparison between stage 1 and the awake state. However, these results are not unexpected given that sleep stages 1 and 2 are transitional stages toward stage 2 and 4 as well as REM respectively.

The $\mathrm{p}$-values derived from the Mann-Whitney-Wilcoxon rank test confirmed the results obtained for the Pearson coefficients (Fig. 5.A). We observed a high discriminative power when deviations from the stationary pattern was considered. About $60 \%$ of the results derived for the difference matrix encounter $p$-values below the $5 \%$ significance level. Even when a Bonferroni correction is employed about $40 \%$ of the results are still significant on a 
$5 \%$ level and about $33 \%$ when a significance level of $1 \%$ is seeked (see inset of Figure $5 . A$ ). At the same time p-values of the SAC-matrix are all above 5\% and only 6 of them encounters values below 0.1 .

The significant results for the difference matrix are primarily observed for the comparison of sleep stage REM with stages 3 and 4 , for the comparisons of stage 1 with sleep stages 2 , 3 and 4 and also the comparisons of stages 2 and 4 provide mostly p-values below 0.05 . In principle, striking similarities of the structure of the average difference matrices as displayed in Figure 5 lead also to notable high p-values for the MWW-rank test. For instance, the comparison of the wake state and stage 1 , or the comparison of sleep stages 3 and 4 lead also to high p-values when magnitudes of the correlations are under consideration.

In the case of the EEG-recordings of the epileptic patients (Figs. 5.C and D) one observes largest discrepancies for the comparison of difference matrices of the pre-seizure and post-seizure period. All Pearson coefficients of this comparison are negative, viz. the average deviations from the stationary pattern are diametrically different, a clear indication of the effect the epileptic activity on the electrical brain dynamics even after seizure termination (Gast et al. 2014, Müller et al. 2011). For the direct comparison of the pre-seizure with the seizure period no consistent result could be derived. In some cases average deviations are positive, in others negative correlated, which could be due to different durations of immediate pre-seizure states in different recordings. For the comparison of the post-seizure with the seizure period Pearson coefficients are dominantly anti-correlated, although also in this case some estimates lead to positive values.

For the comparison of the overall correlations strengths (Fig. 5.C) one observes qualitatively the same results as for the sleep recordings. Average difference matrices lead to more significant difference between phases than correlation matrices themselves. However, now the outcomes are much less significant such that almost none of the comparisons (of the pre- with the post-seizure state) lead to sufficiently small p-values. 
Finally, the comparison of resting states with open and closed eyes lead to some interesting observations (Figs $5 . \mathrm{E}$ and F). First, average distance matrices allow the distinction between the two resting state conditions. In particular for the young adults Pearson correlations between the eyes open and eyes closed condition are dominantly negative. About $15 \%$ of the p-values derived from MWW-rank test are below the $5 \%$ significance level (Bonferroni corrected). For elderly adults the difference is less obvious and $p$-values are not significant, although also in this case the outcomes turn out to be strikingly worse when average correlation matrices are used. However, beside of the distinction of different physiological conditions, also deviations from the stationary pattern seem to be age dependent. As in the case of Figure 4, correlations between the two groups for either condition take notably lower values. If, as suspected above, deviations from the stationary pattern indicate non-stationary dynamical aspects, it could be that not only the stationary pattern but also transient electrical brain activity might evolve with the age. However, given that both groups are measured in different laboratories, these remarks are rather speculative and require confirmation via further studies.

In conclusion, when analyzing deviations from the stationary pattern one obtains a more differentiated picture for dynamical changes of different physiological states than by considering cross-correlations itself. We find remarkable quantitative differences between the results derived for the SAC-matrix compared to those obtained by the difference matrix. Deviations from the stationary pattern provide a highly improved discriminative power for physiologically distinct brain stages. Thus, the results presented in this chapter further indicate that the deviation from the temporarily stable average correlation pattern is a more appropriate measure than correlation pattern itself.

\section{Non-stationary dynamical Aspects of Brain Dynamics}

In Figure 2 we draw the complete time course of all 105 cross-correlation coefficients of subject 9. This figure underpins the quantitative results concerning the stability in time of the stationary pattern. Apparently, most of the correlation coefficients are extremely stable and specifically do not change their signs. Transitions between sleep stages are hardly visible and the time course of the correlation between the SAC-matrix and the 
stationary pattern encounters almost always high values. Thus, as average crosscorrelation result to be (almost) time independent the non-stationary dynamical features we further study whether non-stationary aspects of the brain dynamics is imprinted in the fluctuations of cross-correlations and, furthermore, which coefficients reflect such properties. In order to answer this question one may investigate if there is some relation between the mean value of the correlation coefficients and the size of their fluctuations. To this end, we draw in Figure 6 separately for each sleep stage the size of its 95\% confidence interval versus the mean value of each correlation coefficient.

One observes largest fluctuations and a tendency of lower absolute mean values during wakefulness (see also Fig. 3). The estimates of the widths of the $95 \%$ confidence intervals vary between 0.2 and 1.4 almost over the whole range of the obtained mean values. Note, correlation estimates are restricted within the range $[-1,+1]$ such that confidence intervals are by definition smaller than 2 ; viz. values of the $95 \%$ confidence interval larger than 1 indicate large fluctuations around the stationary pattern. In consequence, no clear dependency between average values and fluctuation strength of the cross-correlation coefficients can be detected for the waking state. Qualitatively the same picture is obtained for sleep stage 1 , although now the size of the fluctuations tend to be smaller.

This picture changes qualitatively when turning to sleep stage 2. Now one observes a clear tendency of the $95 \%$ confidence intervals: correlations coefficients with large dynamical means show smaller fluctuations and vice versa. For estimates of $\left\langle C_{i j}\right\rangle$ close to zero the fluctuations are always larger than 0.4 . On the other hand, very large sizes of the $95 \%$ confidence intervals (values 1.2 or 1.4, like observed in the case of wakefulness or sleep stage 1), are not observed. For sleep stage 2 the confidence intervals are almost always smaller than 0.7 .

This behavior is even more pronounced for the distributions obtained for deep sleep, viz. sleep stages 3 and 4 Similar to sleep stage 2 the size of the average value of correlation coefficients is related to the size of its fluctuations. By trend this is also true for REM-sleep. 
Correlation coefficients with large average values tend to fluctuate less than those with small averages. In particular, those states with higher activation levels like wakefulness, REM sleep and stage 1 , or that which are more susceptible to external stimulation like $\mathrm{W}$ and stage 1, or to endogenous activation and dream like mental activity during REM sleep show by trend higher fluctuations of their correlation coefficients. According to the picture drawn above, this result implies that those correlation coefficients with small average values are stronger involved in non-stationary dynamical aspects of the brain activity than large ones.

\section{Discussion:}

In this article we reported two unexpected observations. First, analyzing EEG recordings we discovered a pronounced correlation pattern of the oscillatory brain activity, which is stable in time. This pattern is generic in the sense that it does not depend on the physiological state, irrespective whether subjects are awake, during light, deep or REM sleep, if they are in different conditions of resting state, or if they encounter a pathological state like an epileptic seizure. The correlation of the temporally resolved SAC-matrix (estimated via a running window approach along the whole night) with the stationary pattern (temporal average over the whole recording), is almost always above 0.85 and only within short moments it drops to values of about 0.6 , which still implies high similarity. Furthermore, this correlation pattern is generic in the sense that it shows an extraordinary similarity across subjects. This affinity between subjects represents a strong indication that this stationary pattern is due to an intrinsic design of a functional network organization, alike the Default Mode Network found in fMRI-studies, but here expressed by the much faster oscillatory electrical brain activity.

Second, we could provide numerical evidence that non-stationary dynamical aspects of the electrical brain activity are more conveniently described by deviations from this stable pattern than by the conventionally used cross-correlations itself. The discriminative power of the difference matrix in comparison to the SAC-matrix is notably higher, such that significance levels may improve by orders of magnitude when deviations are under consideration (see Fig. 5.A). 
We are confident that these observations are not due to technical details of the recording process, particular specifications of the recording equipment, or details of the data preprocessing. In total we considered 72 recordings of 41 subjects in completely different physiological states. Furthermore, the data was acquired by 4 different laboratories in Mexico and Switzerland. Also the chosen EEG reference is not responsible for the observations as could be proven in (Müller et al. 2014). Also volume conduction can be excluded as a possible explanation. Besides, it is hard to believe that volume conduction causes the same effect with the same magnitude in physiological completely different conditions and, furthermore, that volume conduction generates pronounced anticorrelations. In (Müller et al., 2014) it was shown explicitly by estimating maximum lag correlations as well as the weighted phase lag index that the stationary pattern is certainly not due to volume conduction. We repeated such computations for the sleep recordings obtaining similar results (not shown in the Figures). Instead of volume conduction we propose another interpretation of the observed phenomena.

Systems which dissipate energy and interact with its environment like the brain usually do not exploit the whole variety of theoretically possible dynamical states. They prefer to move in phase space within a restricted region, the invariant set or the attractor of the system.

The phase space is the set of all (theoretically) possible dynamical states that the corresponding system may encounter. The dimension of this space is given by the minimal number of variables, which are necessary and sufficient for the complete description of the system, viz. for the determination of all possible observables. For instance, in case of a simple pendulum with friction (energy dissipation) and an external force (interaction with its environment) the minimal number of such variables is two: e.g. the position and the velocity of the pendulum. Knowing these quantities at a given time one is able to calculate all physical observables like e.g. kinetic and potential energy or momentum. Furthermore, the dimension of the phase space equals the number of degrees of freedom of the system. The pendulum, for instance, describes a closed trajectory in a two dimensional phase space called limit cycle. If the external force is switched off, the friction causes that the attractor will be just one point in phase space, a stable fix-point, which resembles the 
resting state of the pendulum. Evidently, more complicated systems may have a much higher dimensional phase space and in the case of brain dynamics this dimension should be an astronomically high number, given that the brain consists of a highly non-trivial network of about $10^{11}$ dynamical units (the neurons) each of them with about $10^{3}$ to $10^{4}$ connections (synapses).

The attractor of a system is invariant in the sense that independent from the starting point of the dynamics in phase space, or equivalently, independent from the kind of external perturbation the system suffers, the dynamics always approaches the same sub-manifold of the phase space (provided that the system stays within a basin of attraction).

For the brain dynamics it is essential that the attractor is not a fix-point. Otherwise, it would have the permanent tendency to switch off and would require permanent energy supply in order to avoid the definite shut down. For the brain it is much more convenient that this invariant set contains all those processes, which are responsible for the maintenance of all vitally indispensable activities, like breathing, control of heart rhythm, temperature control, digestion, etc. However, neuronal circuits controlling such processes are located in evolutionary older brain regions and the spinal cord, the vegetative nerve system, whose electrical activity is not likely to be measured by a scalp recording. Instead, surface EEG capture mainly electrical activity of cortical neurons.

The cortical brain activity shows electric field oscillations on all temporal and spatial scales. This permanent oscillatory behavior is generated by the intrinsic activity of single neurons and extends to the self-organized motion of large neuronal populations (Nunez 1995, 2000). Continuously synchronized neuronal ensembles emerge, persist for a while before they decay, making room for the appearance of other synchronization patterns. In this manner synchronized oscillations of ever changing neuronal populations weave a network of functional relations even between distant cortical regions, such that information transfer and information processing is facilitated. Thus, the hierarchical arrangements of varieties of parallel loops of neural circuits (Buzsaki 2006) get bound and local information processing integrated (Nunez 2000, Tononi et al. 1998, Tononi 2004, 2010). In fact, in order to maintain the brain in an efficient operational mode, a finely tuned balance 
between activation and rest should be adjusted, such that the brain dynamics approximate a critical state close to a second order phase transition (Beggs et al. 2003, Chialvo et al. 2010, Hesse et al. 2014, Poil et al. 2008). This balance is expressed by power laws of the e.g. distribution of spatial sizes of synchronization pattern as well as the distribution of live times of synchronized neuronal populations (Eguiluz et al. 2005, Linkenkaer-Hansen et al. 2001, Miller et al. 2009, Poil et al. 2008). In this regime the brain produces a maximal variety of different synchronization patterns, while simultaneously spatial correlation encounters largest length. In this sense, the attractor dynamics would be a kind of dynamical ground state.

Our results show that this variety of synchronized space-time structures are not randomly distributed over the scalp but obey some global order principles such that a temporarily stable scaffold of pronounced cross-correlations emerge. In this way, a specific, task related synchronization pattern does not require to be created within a tissue of almost silent neurons. Rather, already existing space-time interrelations merely have to be modulated. Hence, if the enduring neural background activity can be related to the attractor of the brain dynamics, task related actions or activity provoked by external stimuli are expressed by a specific adjustment of a permanently existent oscillatory mode. Thus, higher order processes like cognitive functions, motor control or sensory processing are expressed by deviations from the attractor mode. Taking into account that we are almost permanently subject to external stimuli this implies that in fact the brain is almost permanently located on transient states, such that the system fluctuates continuously around the attractor in phase space.

This picture is further supported by the brains energy expenditure. Considering that the human brain represents about $2 \%$ of the whole body weight its energy consumption with about $20 \%$ of the total energy budget is notably high (Raichle et al. 2006). However, this number is independent of the brain's activity and stays rather stable. It was estimated that the local increase of the energy consumption related to the processing of external stimuli varies between $0.5 \%$ to $2 \%$ (Raichle et al,. 2006a and 2006b), a surprisingly low value. Even the fact that only $10 \%$ of the synapses carry information of the external world (Peters et al. 1994) does not fully explain such small variations of the energy consumption. However, 
the scenario described above may provide a consistent explanation. If the resting state activity is characterized by cortical standing waves of the electric field (Nunez 2000, Müller et al. 2014), generated by a continuous alternation of the number of active synapses per unit volume (Nunez 2000), and the response to demands of the external world merely consists in the modulation of this oscillating mode, one does not expect a huge increase of the energy consumption. Instead, it is plausible that the majority of the energy is used by the attractor activity itself, viz. the maintenance of the oscillatory neural background activity.

If this scenario resembles a realistic picture, the attractor should imprint a characteristic correlation pattern on the average cross-correlation matrix as observed in this contribution, which should be stable in time because fluctuations around the attractor cancel out when averages are taken over long time intervals.

We proved the last statement via some model calculations (see supplementary material), where we derived correlation matrices from different types of non-stationary dynamical systems. In a first case we investigate a system, which moves almost permanently on transients around a stable attractor. For this system it turns out that deviations from the attractor-mode, which manifest as deviations from the unperturbed correlation matrix, cancel out by the time-average, such that both correlation matrices are also quantitatively extremely similar ( $C=0.98, p$-value $=0.346$, derived from MWW rank test). A situation which resembles the findings of the average of the correlation matrices derived from the EEG-recordings.

For the second system we continuously deformed the attractor geometry along the time series. Then we compared the average correlation matrices from the first and second half of the derived time series obtaining striking differences between the average correlation patterns $(C=0.26$, $p$-value=0.038). By deforming the attractor geometry the crosscorrelation structure change qualitatively in time, a situation which definitely do not reflect the observations reported above. 
Note, if in both model calculations the information about the deterministic structure is destroyed, e.g. via Iterative Amplitude Adjusted Fourier Transform Surrogates (Schreiber Schmitz, 2000), all cross correlations are destroyed and the distribution of $p$-values follow closely the null hypothesis generated by white noise. Key-point of these surrogates is, that amplitude distribution as well as the Power spectrum of the signals are conserved, while Fourier phases are substituted by random numbers, uniformly distributed between zero and $2 \pi$. However, if random phases are chosen such that phase differences between signals are maintained, deterministic features are destroyed while cross-correlations (as well as nonlinear interrelationships between signals) are conserved also. In this case crosscorrelations estimated from surrogate data approximate those estimated by the EEG-data.

Hence, the first scenario is consistent with what one observes analyzing empirical real world data. The result of such averages leads to the observation of a pronounced, stable correlation pattern, which seems to be independent of the physiological state of the brain. Independently if one considers the sleeping brain in different sleep stages, the brain in the awake state, different conditions of resting state or drastic pathologic activity like an epileptic seizure, the topology of spatial correlations as well as the overall average correlations result to be extremely stable and statistically indistinguishable.

Finally, such rigid skeleton of large scale spatial correlations may result beneficial for the orchestration of segregated local functional networks and could provide an efficient mechanism for the integration of local information processing. Waves are entities that implement spatial-temporal structures and large scale correlation, as observed in the stationary pattern, which allow a fast communication even between distant areas. This oscillatory mode provide a convenient framework for the generation of bottom-up and top-down processes, where local excitations may rapidly propagate due to large scale correlations provoking global modulations of the standing waves, and, on the other hand, local perturbations might be suppressed by a globally collective dynamics.

Note, these findings do not imply that brain activity is a static monolithic process (as proven by the model calculations presented in the supplementary material) and the present study does not challenge the highly non-stationary character of EEG signals. But it 
provides strong evidence for the existence of an attractor of the brain dynamics, a picture which is consistent with abundant literature about the energy consumption and that portrays the brain as a complex system close to a second order phase transition, a situation most favorable to maintain the brain in an efficient operational mode and transient dynamics provoke excursions in phase space around the attractor.

Hence, if non-stationary dynamical aspects of the brain dynamics are imprinted in transient behavior in phase space, the quantification of deviations from the stationary correlation structure constitutes a more appropriate strategy of analysis than conventional concepts, providing a higher significance level of numerical estimates and a more consistent picture across subjects. So far, we reported solely absolute deviations of broad band signals, averaged over the whole scalp. However, in order to get an improved description of non-stationary dynamical aspects one needs to look in more detail to the spatial distribution of the deviations from the stationary pattern in a time resolved manner separately for different frequency bands. As shown in the present work, the pattern of deviations are qualitatively different for e.g. different sleep stages, that is, corresponding difference matrices are located toward different directions in matrix space, which implies that a qualitative different behavior should be expected for different sleep stages.

Figure 6 documents that preferentially those matrix elements with low average values show dominant fluctuations and, in line with the interpretation of a transient movement around the attractor activity, in particular these matrix elements carry relevant information about the dynamics of non-stationary dynamical features like higher order cognitive processes or reaction to external stimuli. Although it remains to study which brain areas are dominantly affected by such changes and under which conditions, the present findings already imply that any kind of thresholding, where matrix elements with small cross-correlation values are principally excluded from the analysis, constitute an unfavorable strategy. If, as in many studies, matrix elements below e.g. 0.3 or 0.4 are systematically discarded, essential dynamical features might be missed in general.

Another approach, which might offer a complementary view, follows a similar philosophy than the traditional Principal Component Analysis. If the EEG recordings are projected to 
the eigenbasis of the stationary pattern, the transformed datasets do no longer contain the observed temporarily stable correlation pattern. Hence, nonzero elements of the SACmatrices constructed from signals transformed this way also indicate deviations from the stationary pattern.

\section{Conclusions:}

The stationary correlation pattern identified in the present study is consistent with the attractor dynamics of the brain activity, which contains the minimal set of vitally indispensable activities and maintains the cerebral cortex in an efficient dynamical mode. In this picture, higher order processes, like e.g. cognitive acts or different physiological states (as e.g. different sleep stages) are conveniently expressed by deviations from the stationary pattern. We provided numerical evidence that in comparison to traditional concepts discriminations becomes higher when derivations from the stable correlation pattern are studied.

The observations presented in this paper lead to a consistent picture in terms of dynamical system theory and could open new avenues for brain-signal-analysis, which allow to illuminate novel aspects of the brain dynamics.

\section{Acknowledgment}

This work was supported by CONACyT Mexico, Proj. No. CB-156667. CR received support from the Swiss League Against Epilepsy. Recordings of the elderly subjects during rest have been obtained by Mtra. Josefina Montes Rojas and Dr. Estañol Vidal from the Instituto Nacional de Ciencias Médicas y Nutrición Salvador Zubirán. 
References:

Achermann P, Borbély AA. (1998). Coherence analysis of the human sleep electroencephalogram. Neuroscience 85:1195-120.

Achermann, P., Rusterholz, T., Dürr, R., König, T., \& Tarokh, L. (2016). Global field synchronization reveals rapid eye movement sleep as most synchronized brain state in the human EEG.Royal Society Open Science,3(10), 160201.

Allen EA, Damaraju E, Plis SM, Erhardt EB, Eichele T, Calhoun VD., 2014., Tracking WholeBrain Connectivity Dynamics in the Resting State, Cereb. Cortex; 24, 663-676

Allen EA, Damaraju E, Eichele T, Wu L, Calhoun VD, 2018, EEG Signatures of Dynamic Functional Network Connectivity States, Brain Topogr; 31, 101-116

Ansari-AsI K, Senhadji L, Bellanger JJ, Wendling F. (2006) Quantitative evaluation of linear and nonlinear methods characterizing interdependencies between brain signals. Phys Rev E Stat Nonlin. Soft Matter Phys;74:031916.

Barry RJ, Clarke AR, Johnstone SJ, Magee CA, Rushby JA. 2007, EEG differences between eyes-closed and eyes-open resting conditions, Clin. Neurophysiol; 118: 2765-2773

Bartolomei F, Wendling F, Vignal J-P, Chauvel P, Liégeois-Chauvel C. (2002). Neural networks underlying epileptic humming. Epilepsia 43:1001-1012.

Bartolomei F, Wendling F, Régis J, Gavaret M, Guye M, Chauvel P. (2004). Pre-ictal synchronicity in limbic networks of mesial temporal lobe epilepsy. Epilepsy Res 61:89-104.

Beggs, J. M. \& Plenz, D. (2003). Neuronal avalanches in neocortical circuits. The Journal of Neuroscience 23, 11167-11177.

Boccaletti S, Latora V, Moreno Y, Chavez M, Hwang D-U, (2006) Complex networks: Structure and dynamics, Physics Reports 424: 175 - 308. 
Buckner RL, Andrews-Hanna JR, Schacter DL. (2008). The brain's default network: anatomy, function, and relevance to disease. Ann. N Y Acad Sci 1124:1-38.

Buzsaki G. Rhythms of the Brain, Oxford University Press, 2006.

Cantero, J. L., Atienza, M., \& Salas, R. M. (2000). State-modulation of cortico-cortical connections underlying normal EEG alpha variants. Physiology \& behavior, 71(1-2), 107115.

Chialvo, D. R. (2010). Emergent complex neural dynamics. Nature Physics 6, 744-750.

Corsi-Cabrera M, Meneses S, Molina E (1987) Correlación Interhemispherica y Acoplamiento Temporal de la Actividad Eléctrica Cortical Durante la Vigilia, la Etapa II y el Sueño Paradójico en el Hombre. Rev Mex Psicol 4:100-108.

Corsi-Cabrera, M, Solis-Ortiz, S., Guevara, M.A. (1997). Stability of EEG inter-and intrahemispheric correlation in women. Electroencephalography and clinical Neurophysiology 102, 248-25.

Corsi-Cabrera M, Miró E, del-Río-Portilla Y, Pérez-Garci E, Villanueva Y, Guevara MA (2003) Rapid eye movement sleep dreaming is characterized by uncoupled EEG activity between frontal and perceptual cortical regions. Brain Cogn 51:337-345.

Corsi-Cabrera, M., Galindo-Vilchis, L., del-Río-Portilla, Y., Arce, C., Ramos-Loyo, J. (2007). Within-subject reliability and inter-session stability of EEG power and coherent activity in women evaluated monthly over nine months. Clinical Neurophysiology 118, 9-21.

Damaraju E, Allen EA, Belger A, Ford JM, McEwen S, Mathalon DH, Mueller BA, Pearlson GD, Potkin SG, Preda A, Turner JA, Vaidya JG, van Erp TG, Calhoun VD, 2014, Dynamic functional connectivity analysis reveals transient states of dysconnectivity in schizophrenia, Neurolmage: Clin.; 5, 298-308

De Gennaro, L., Ferrara, M., Curcio, G., \& Cristiani, R. (2001). Antero-posterior EEG changes during the wakefulness-sleep transition. Clinical neurophysiology,112(10), 1901-1911. 
Duckrow RB, Zaveri HP (2005) Coherence of the electroencephalogram during the first sleep cycle. Clin Neurophysiol 116:1088-1095.

Eguiluz V.M, Chialvo D.R., Cecchi G.A., Baliki M., Apkarian A.V. (2005). Scale free brain functional networks, Phys. Rev. Lett. 94: 018102.

Engel AK, Singer W (2001). Temporal binding and the neural correlates of sensory awareness. Trends Cogn Scie;5:16-25.

Fox MD, Snyder AZ, Vincent JL, Corbetta M, Van Essen DC, Raichle ME. (2005). The human brain is intrinsically organized into dynamic, anti-correlated functional networks. Proc Natl Acad Sci U S A 102:9673-9678.

Fries P (2005) A mechanism for cognitive dynamics: neuronal communication through neuronal coherence. Trends Cogn Sci 9:474-480.

Galka A. Topics in Nonlinear Time Series Analysis: With Implications for EEG Analysis, Advanced Series in Nonlinear Dynamics, Volume 14, Wolrd Scientific, 2000.

Gast H, Müller M, Rummel C, Roth C, Mathis J, Schindler K, Basetti CL (2014). Epileptic seizures as condensed sleep: an analysis of network dynamics from electroencephalogram signals. J. Sleep Res. 23: 268-73.

Greicius M.D., Krasnow B, Reiss AL, Menon V. (2003). Functional connectivity in the resting brain: a network analysis of the default mode hypothesis. Proc Natl Acad Sci U S A 100: 253-258.

Greicius M. (2008). Resting-state functional connectivity in neuropsychiatric disorders. Curr Opin Neurol 21:424-430.

Guevara, M.A., Lorenzo, I., Arce, C., Ramos, J., Corsi-Cabrera, M., (1995). Inter- and intrahemispheric EEG correlation during sleep and wakefulness. Sleep 18, 257-265. 
Guye M, Régis J, Tamura M, Wendling F, Mc Gonigal A, Chauvel P, Bartolomei F. 2006. The role of corticothalamic coupling in human temporal lobe epilepsy. Brain 129: 1917-1928.

He B.J., Snyder AZ, Zempel JM, Smyth MD, Raichle ME. 2008. Electrophysiological correlates of the brain's intrinsic large-scale functional architecture. Proc Natl Acad Sci USA 105:16039-16044.

Hesse J, Gross T, (2014) Self-organized criticality as a fundamental property of neural systems, Front. Syst. Neurosci. 8.

Honey CJ, Sporns O, Cammoun L, Gigandet X, Thiran JP, Meuli R, Hagman P. (2009). Predicting human resting-state functional connectivity from structural connectivity. Proc Natl Acad Sci USA 106:2035-2040.

Jann K, Kottlow M, Dierks T, Boesch C, Koenig T. (2010). Topographic electrophysiological signatures of $\mathrm{fMRI}$ resting state networks. PLoS One 5:e12945.

Kaminski M, Blinowska KJ, Szclenberger W (1997) Topographic analysis of coherence and propagation of EEG activity during sleep and wakefulness. Electroecephalogr Clin Neurophysiol 102:216-227.

Kantz, H., \& Schreiber, T. (2004). Nonlinear time series analysis (Vol. 7). Cambridge University Press.

Kaur M, Prakash NR, Kalra P, Puri GD, 2917, EEG Signals During Eyes-Open and Eyes Closed State, IJCESR, 4: 2394-0697

Keener, J. P., \& Sneyd, J. (1998). Mathematical physiology (Vol. 1). New York: Springer.

Kramer MA, Eden UT, Lepage KQ, Kolaczyk ED, Bianchi MT, Cash SS. 2011. Emergence of persistent networks in long-term intracianial EEG recordings. J Neurosci 31:15757-15767.

Kreuz T, Mormann F, Andrzejak RG, et al. (2007) Measuring synchronization in coupled model systems: a comparison of different approaches. Physica D;225:29-42. 
Laloux L, Cizeau P, Bouchaud J-P, Potters M (1999). Noise Dressing of Financial Correlation Matrices, Phys. Rev. Lett. 83, 1467-1470.

Lesser, R. P. (1986). Guideline seven: A proposal for standard montages to be used in clinical EEG. Journal of Clinical Neurophysiology, 3(3 SUPPL. 1), 26-33.

Linkenkaer-Hansen K., Nikouline V.V., Palva J.M. Ilmoniemi R.J. (2001). Long-Range Temporal Correlations and Scaling Behavior in Human Brain Oscillations, The J. of Neurosci. 21: 1370-1377.

Llinás, R., Ribary, U., (1993). Coherent 40-Hz oscillation characterizes dream state in humans. Proc. Natl. Acad. Sci. U. S. A. 90, 2078-2081.

Marín García AO, Müller MF, Schindler K, Rummel C (2013). Genuine cross-correlations: Which surrogate based measure reproduces analytical results best? Neural Networks 46: 154-164.

Miller K.J., Sorensen L.B., Ojemann J.G., den Nijs M., (2009). Power-Law Scaling in the Brain Surface Electrical Potential, PlozS Comp. Biol. 5(12): e1000609.

Mormann F, Kreuz T, Rieke C, Andrzejak RG, Kraskov A, David P, Elger CE, Lehnertz, 2005, On the predictability of epileptic seizures, Clin. Neurophysiol.; 116, 569-587

Müller MF, Baier G, López Jiménez Y, Marín García AO, Rummel C, Schindler K. (2011). Evolution of genuine cross-correlation strength of focal onset seizures. J Clin Neurophysiol 28:450-462.

Müller M.F., Rummel C, Goodfellow M, Schindler K (2014). Standing Waves as an Explanation for Generic Stationary Correlation Patterns in Noninvasive EEG of Focal Onset Seizures, Brain Connectivity, 4: 131-144.

Netoff T.I., Schiff SJ. (2002). Decreased neural synchronization during experimental seizures. J Neurosci. 22:7297-7307. 
Nyberg L, McIntosh AC, Cabeza R, Nilsson LG, Houle S, Habib R, Tulving E. (1996). Network analysis of positron emission tomography regional cerebral blood flow data: ensemble inhibition during episodic memory retrieval. J Neurosci 16: 3753-3759.

Nielsen, T., Abel, A., Lorrain, D., Montplaisir, J., 1990. Interhemispheric EEG coherence during sleep and wakefulness in left- and right-handed subjects. Brain Cogn. 14, 113-125.

Nunez P.L. Neocortical Dynamics and Human EEG Rhythms. New York: Oxford University Press (1995).

Nunez P.L. (2000). Toward a quantitative description of large-scale neocortical dynamic function and EEG. Behav Brain Sci 23:371-437.

Pereda E, Quian Quiroga R, Bhattacharya J (2005). Nonlinear multivariate analysis of neurophysiological signals, Prog in Neurobiol. 77, 1-37.

Pérez-Garci, E., del Río-Portilla, Y., Guevara, M.A., Arce, C., Corsi-Cabrera, M., 2001. Paradoxical sleep is characterized by uncoupled gamma activity between frontal and perceptual cortical regions. Sleep 24, 118-126.

Peters A., Payne BR, Budd J, (1994). A Numerical Analysis of the Geniculocortical Input to Striate Cortex in the MonkeyCereb. Cortex 4, 215.

Plerou V., Gopikrishnan P, Rosenow B, Nunes Amaral LA, Stanley HE (1999), Universal and Nonuniversal Properties of Cross Correlations in Financial Time Series, Phys. Rev. Lett. 83: 1471-1474.

Poil S-S., van Ooyen A, Linkenkaer-Hansen K, (2008) Avalanche dynamics of human brain oscillations: relation to critical branching processes and temporal correlations, Human Brain Mapping, 29: 770-777.

Raichle ME, MacLeod AM, Snyder AZ, Powers WJ, Gusnard DA, Shulman GL. (2001). A default mode of brain function. Proc Natl Acad Sci U S A 98:676-682. 
Raichle ME, Mintun M. (2006). Brain work and brain imaging. Annu Rev Neurosci 29:449476.

Raichle ME. (2006)a. The brains dark energy. Science 314:1249-1250.

Raichle ME., Mintun M.A. (2006)b. Brain Work and Brain Imaging, Annu. Rev. Neurosci. 29: 449-476.

Rechtschaffen, A., Kales, A. (Eds.), 1968. A Manual of Standardized Terminology, Techniques

and Scoring System for Sleep Stages of Human Subjects. Brain Information Service, Brain Research Institute, University of California, Los Angeles.

Rummel C, Müller M, Baier G, Amor F, Schindler K (2010). Analyzing spatio-temporal patterns of genuine cross-correlations, J. Neurosci. Meth. 191: 94-100.

Schindler K, Leung H, Elger CE, Lehnertz K. 2006. Assessing seizure dynamics by analysing the correlation structure of multichannel intracranial EEG. Brain 130:65-77.

Schindler K, Bialonski S, Horstmann M-T, Elger CE, Lehnertz K. 2008. Evolving functional network properties and synchronizability during human epileptic seizures. Chaos 18:033119.

Schreiber T, Schmitz A, 2000, Surrogate Time Series, Phys. Nonlin. Phenom; 142: 346-381

Shulman G, Fiez J, Corbetta M, Buckner R, Miezin R, Raichle M, Peterson S. (1997). Common blood flow changes across visual tasks: II. Decrease in cerebral cortex. J Cogn Neurosci 9:648-663.

Singer, W. (1996). Neuronal synchronization: A solution to the binding problem. The mindbrain continuum: Sensory processes, 101-130.

Singer, W., 1999. Neural synchrony: A versatile code for the definition of relations? Neuron 24, 49-65. 
Steriade, M., 1997. Synchronized activities of coupled oscillators in the cerebral cortex and thalamus at different levels of vigilance. Cereb. Cortex 7, 583-604.

Steriade M, McCarley RW. Brainstem control of wakefulness and sleep. New York: Plenum Press, 1990.

Tan B, Kong X, Yang P, Jin Z, Li L. 2013, The Difference of Brain Functional Connectivity between Eyes-Closed and Eyes-Open Using Graph Theoretical Analysis, Comp. and Math. Meth. in Med, 976365

Tononi G, Edelman GM. (1998). Consciousness and complexity. Science 282:1846-1851.

Tononi G, (2004). An information integration theory of consciousness, BMC Neurosci 5:

Tononi, G., (2010). Information integration: its relevance to brain function and consciousness. Arch. Ital. Biol. 148, 299-322.

Varela, F., Lachaux, J. P., Rodriguez, E., \& Martinerie, J. (2001). The brainweb: phase synchronization and large-scale integration. Nature reviews neuroscience, 2(4), 229.

Voss U, Holzmann R, Tuin I, Hobson JA (2009) Lucid dreaming: a state of consciousness with features of both waking and non-lucid dreaming. Sleep 32:1191-1200.

Wendling F, Bartolomei F, Bellanger JJ, Bourien J, Chauvel P. 2003. Epileptic fast intracerebral EEG activity: evidence for spatial decorrelation at seizure onset. Brain 126:1449-1459.

Williams, R., Karakan, I., Hursch, C.J. Electroencephalography (EEG) of human sleep: Clinical applications. John Wiley and Sons, inc. 1974. USA.

Zou Q, Yan B-K, Gu H, Liu D, Wang DJJ, Gao J-H, Yang Y, Zang, Y-F. 2015, Detecting Static and Dynamic Differences between Eyes-Closed and Eyes-Open Resting State using ASL and BOLD fMRI, PLOS ONE 10, e0121757 
TABLE 1: Information about the 10 male subjects of the sleep recordings. Shown is the age and the percentage of the recording each subject stayed in the unambiguously identified awake or a particular sleep stage.

\begin{tabular}{|c|c|c|c|c|c|c|c|}
\hline Subject & Age & $\%$ wake & $\begin{array}{c}\text { \% NREM } \\
1\end{array}$ & $\begin{array}{c}\text { \% NREM } \\
2\end{array}$ & $\begin{array}{c}\text { \% NREM } \\
3\end{array}$ & $\begin{array}{c}\text { \% NREM } \\
4\end{array}$ & $\%$ REM \\
\hline 1 & 21 & 0.9 & 2.4 & 51.9 & 4.2 & 15.8 & 24.9 \\
\hline 2 & 25 & 9.2 & 4.7 & 45.0 & 6.6 & 14.2 & 20.3 \\
\hline 3 & 27 & 5.0 & 1.6 & 47.4 & 5.0 & 16.9 & 24.2 \\
\hline 4 & 21 & 5.8 & 5.2 & 50.9 & 6.0 & 8.6 & 23.5 \\
\hline 5 & 24 & 2.9 & 2.5 & 49.1 & 6.8 & 16.9 & 21.9 \\
\hline 6 & 29 & 2.4 & 3.3 & 53.4 & 4.9 & 15.8 & 20.2 \\
\hline 7 & 24 & 0.7 & 2.7 & 52.5 & 6.5 & 17.5 & 20.1 \\
\hline 8 & 26 & 0.6 & 3.5 & 61.5 & 4.5 & 9.0 & 20.9 \\
\hline 9 & 31 & 6.0 & 2.8 & 38.9 & 9.0 & 24.0 & 19.2 \\
\hline 10 & 24 & 7.0 & 3.9 & 43.6 & 8.9 & 22.1 & 14.5 \\
\hline
\end{tabular}


Table 2: Information about sex, age and seizure duration of 20 peri-ictal recordings from 9 epilepsy patients.

\begin{tabular}{|c|c|c|c|c|}
\hline Patient & Age & Sex & Seizure & Duration/sec. \\
\hline \multirow{3}{*}{1} & \multirow{3}{*}{22} & \multirow{3}{*}{$\mathrm{m}$} & 1 & 96 \\
\hline & & & 2 & 122 \\
\hline & & & 3 & 84 \\
\hline \multirow[t]{2}{*}{2} & \multirow[t]{2}{*}{28} & \multirow[t]{2}{*}{$f$} & 4 & 132 \\
\hline & & & 5 & 204 \\
\hline \multirow[t]{2}{*}{3} & \multirow[t]{2}{*}{45} & \multirow[t]{2}{*}{$f$} & 6 & 113 \\
\hline & & & 7 & 52 \\
\hline \multirow{3}{*}{4} & \multirow{3}{*}{23} & \multirow{3}{*}{$\mathrm{m}$} & 8 & 150 \\
\hline & & & 9 & 120 \\
\hline & & & 10 & 203 \\
\hline \multirow[t]{2}{*}{5} & \multirow[t]{2}{*}{33} & \multirow[t]{2}{*}{$\mathrm{m}$} & 11 & 56 \\
\hline & & & 12 & 10 \\
\hline \multirow{3}{*}{6} & \multirow{3}{*}{18} & \multirow{3}{*}{$\mathrm{m}$} & 13 & 40 \\
\hline & & & 14 & 54 \\
\hline & & & 15 & 119 \\
\hline \multirow{3}{*}{7} & \multirow{3}{*}{27} & \multirow{3}{*}{$f$} & 16 & 91 \\
\hline & & & 17 & 94 \\
\hline & & & 18 & 50 \\
\hline
\end{tabular}


Page 44 of 69

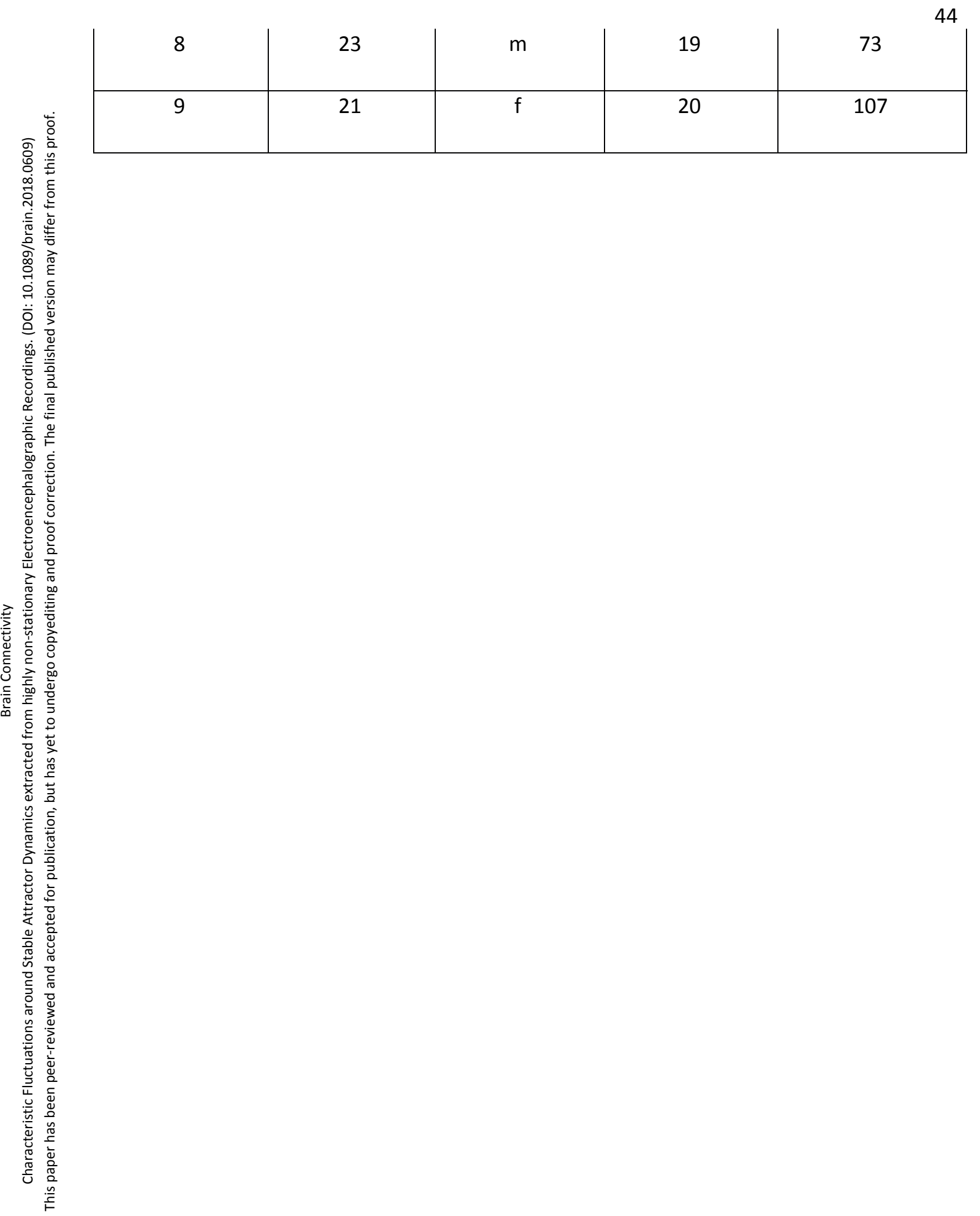


Table 3: Information about sex, age from 21 healthy subjects in resting state

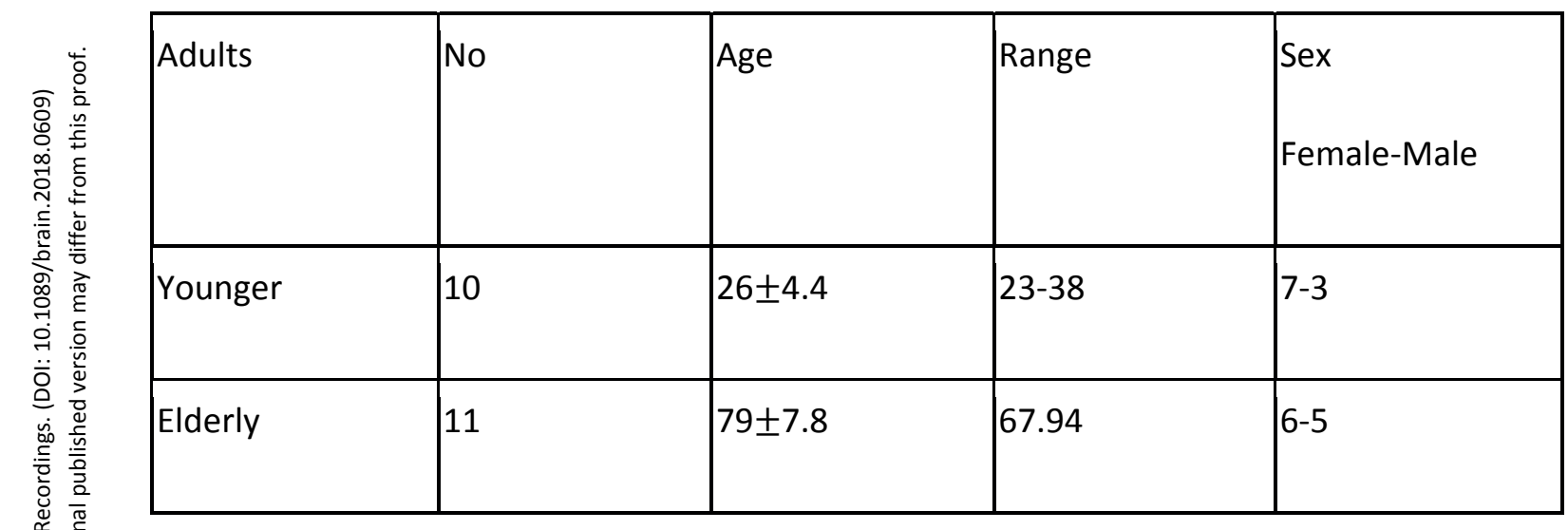


Figures Captions

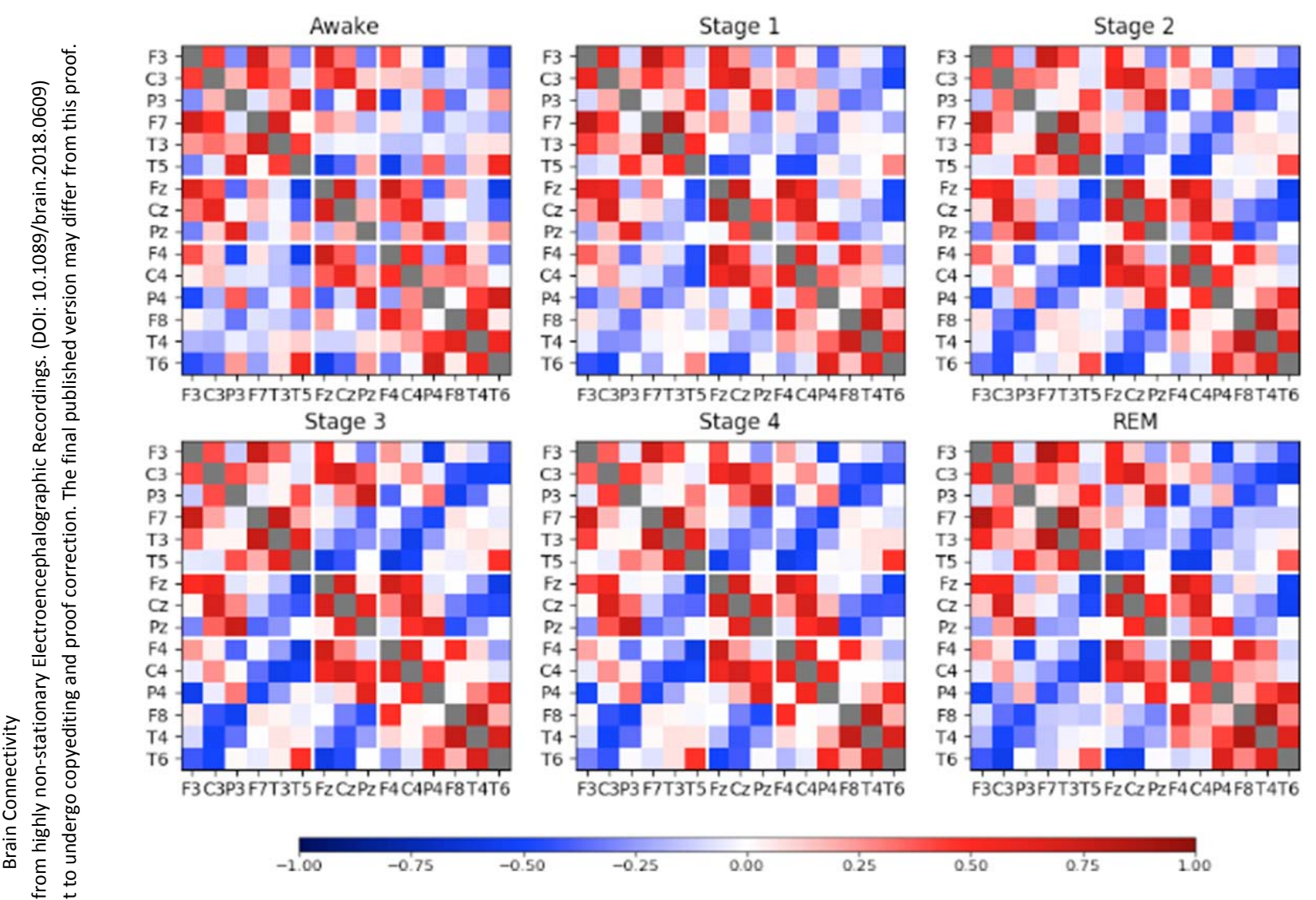

Figure 1: Average correlation-matrices of subject 9 of Table 1. Each average is taken over all data segments belonging to a particular sleep stage. 


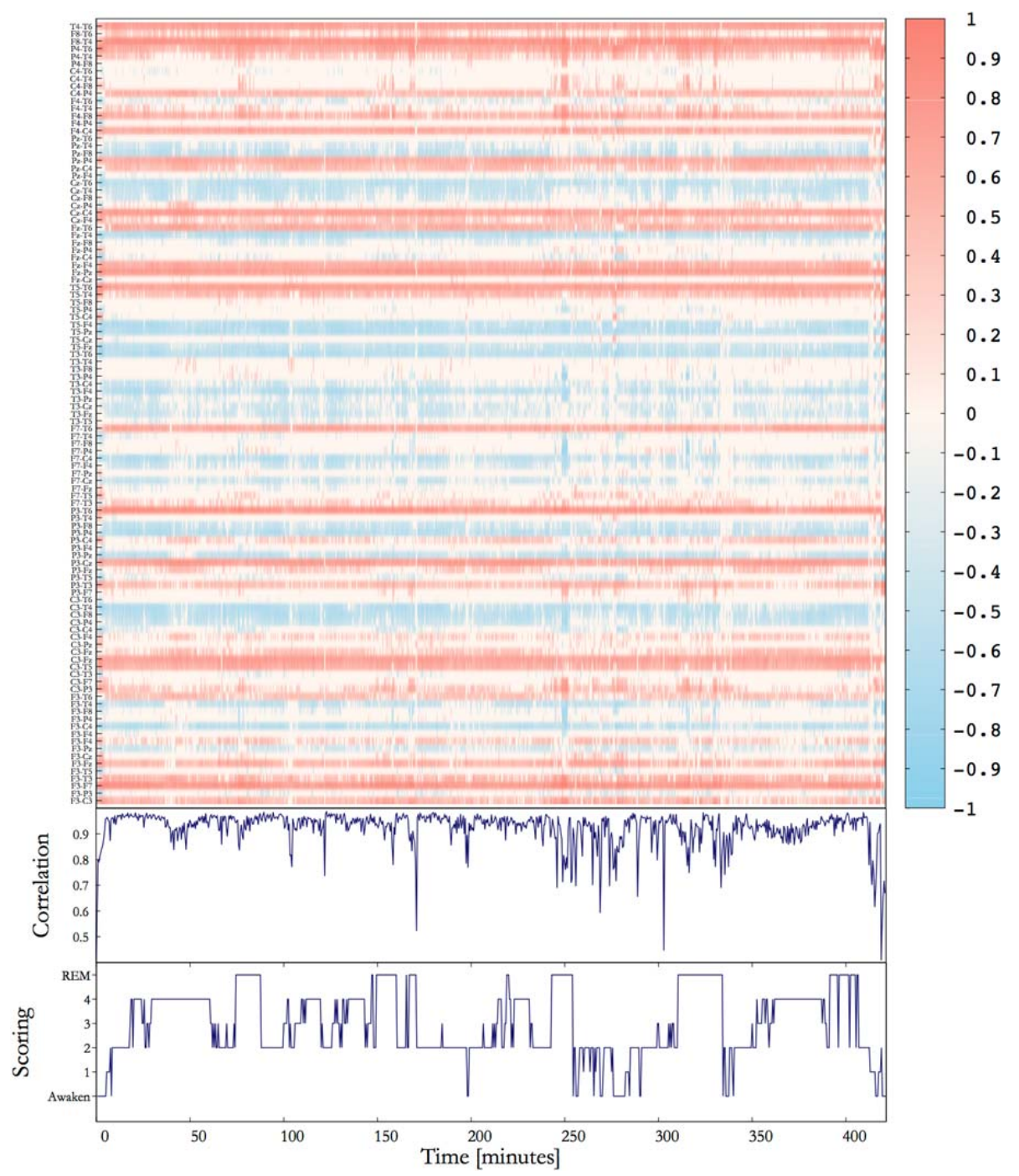

Figure 2: Case study of subject 9. Upper panel: Time evolution of all 105 correlation coefficients of the SAC matrix. Middle panel: Correlation between the time dependent matrix vectors formed from the SAC-matrix and the stationary pattern. Lower panel: Scoring of the corresponding polysomnography. 


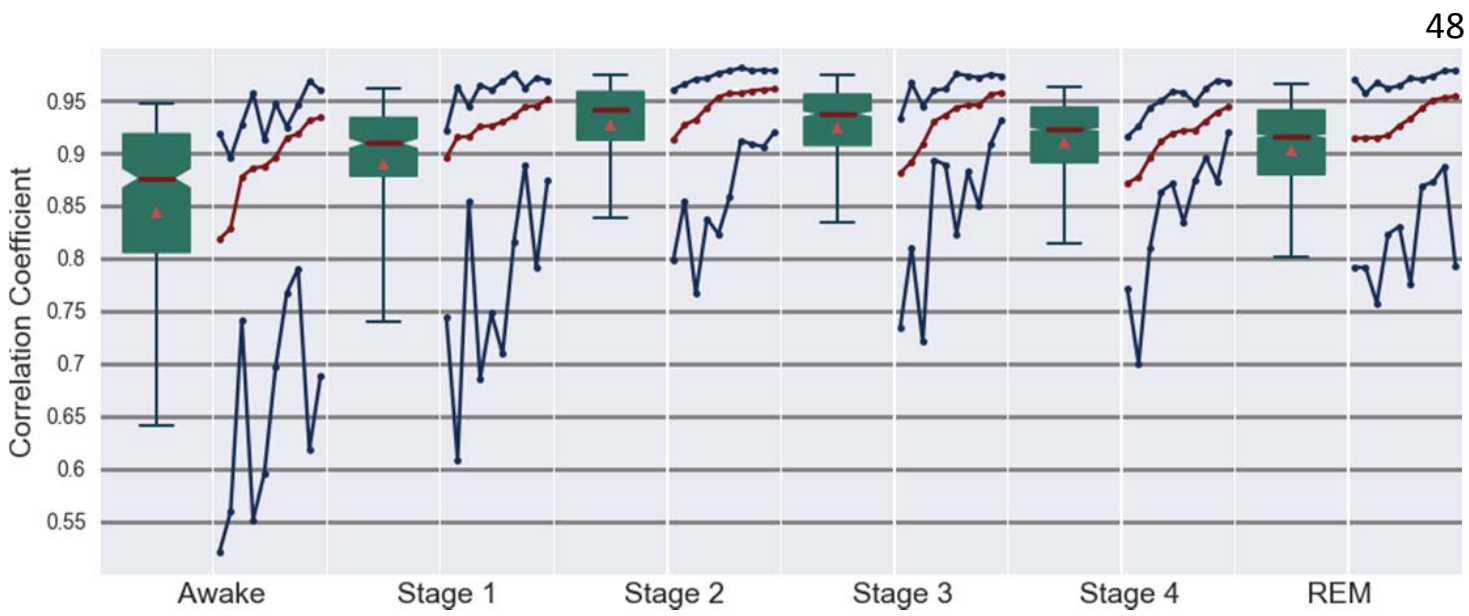

Figure 3: Summary statistics over all 10 subjects of Table 1. For each sleep stage two columns are drawn. The box plot on the left shows median value and the borders of the $50 \%$ and $90 \%$ confidence interval of the Pearson Correlations estimated between the SACmatrix of a given data window and the stationary pattern. Furthermore, as a red triangle the average value is shown. The right column shows median (red color) and the borders of the $90 \%$ confidence interval (blue color) separately for the 10 healthy subjects, ordered by ascending median correlation. Solid lines are just or guidance of the eye. 


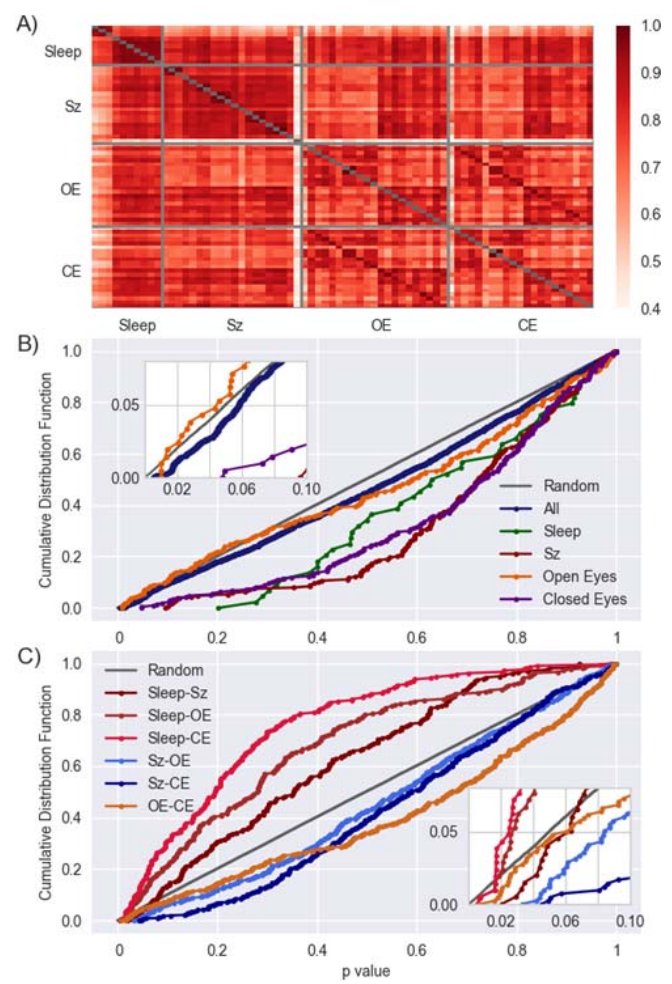

Figure 4: A) Pearson coefficients for the pairwise comparison stationary pattern. Considered are sleep recordings (Sleep), the peri-ictal average of focal onset seizures (SZ), Resting state for open eyes (OE) and closed eyes (CE) for young and elderly adults. Young adults are the first half of the resting state recordings, elderly adults the second half.

B) Cumulative distributions of $p$-values derived by the non-parametric Mann-WhitneyWilcoxon-rank test for the pairwise comparison of the stationary pattern of all recordings (blue symbols), the 10 sleep recordings (green), the 20 peri-ictal averages of temporal lobe seizures (brown), open (orange) and closed eyes (purple). As a reference we also draw the results of 100000 samples of Gaussian white noise with the same size as the empirical data (gray shaded squares). The inset shows the left tail of the distributions C) Same as B but now the stationary pattern of sleep EEGs is compared with the results for seizure EEGs (dark brown), open eyes (light brown) and closed eyes condition (red). Furthermore Seizure data is compared to open (light blue) and closed eyes condition (dark blue) and finally a pairwise comparison with open and closed eyes (orange) is shown. 

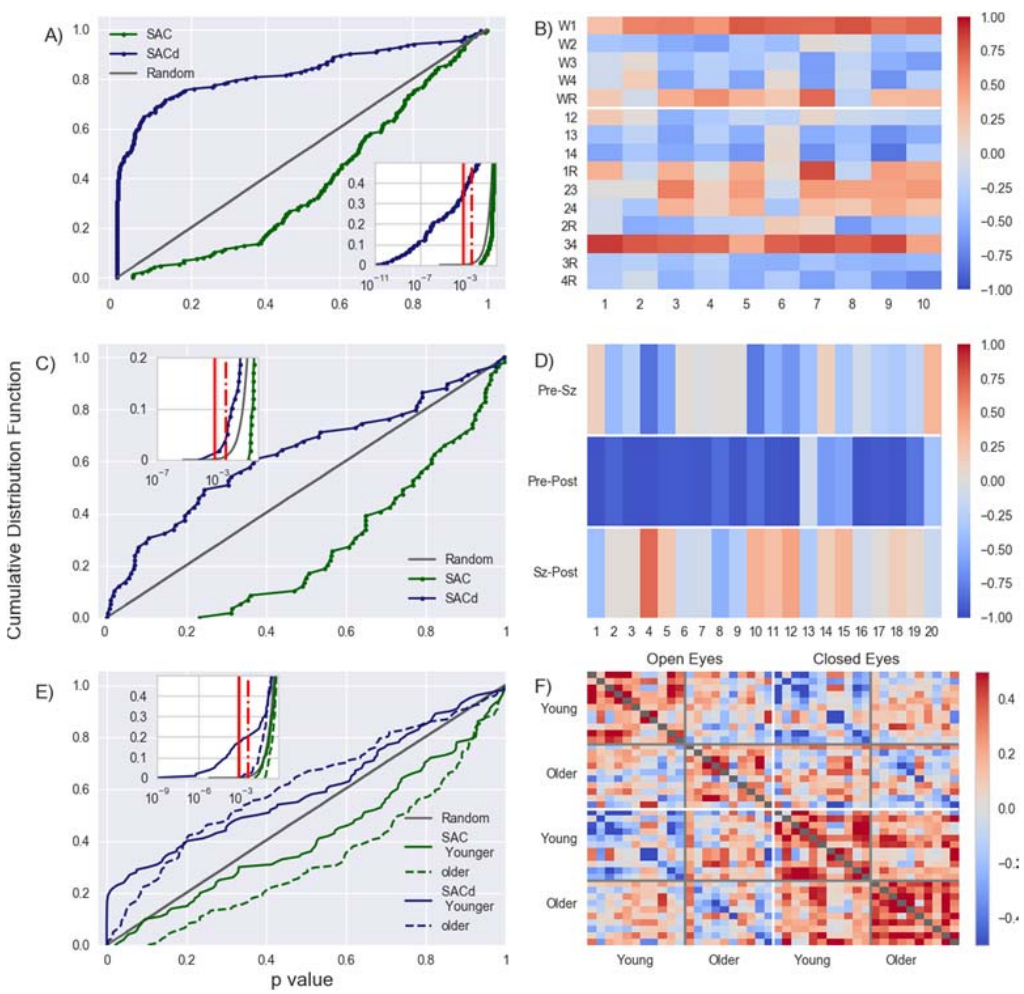

Figure 5: A and B Quantitative comparison of the stationary pattern derived for different sleep stages, $C$ and $D$ for the pre-seizure, seizure and the post-seizure period, and $E$ and $F$ for the open and closed eye condition of young and elderly adults. Figures $A, C$ and $E$ display the cumulative distribution of $p$-values derived from the Mann-Whitney-Wilcoxonrank test, Figures $\mathrm{B}, \mathrm{D}$ and $\mathrm{F}$ the corresponding Pearson coefficients. 

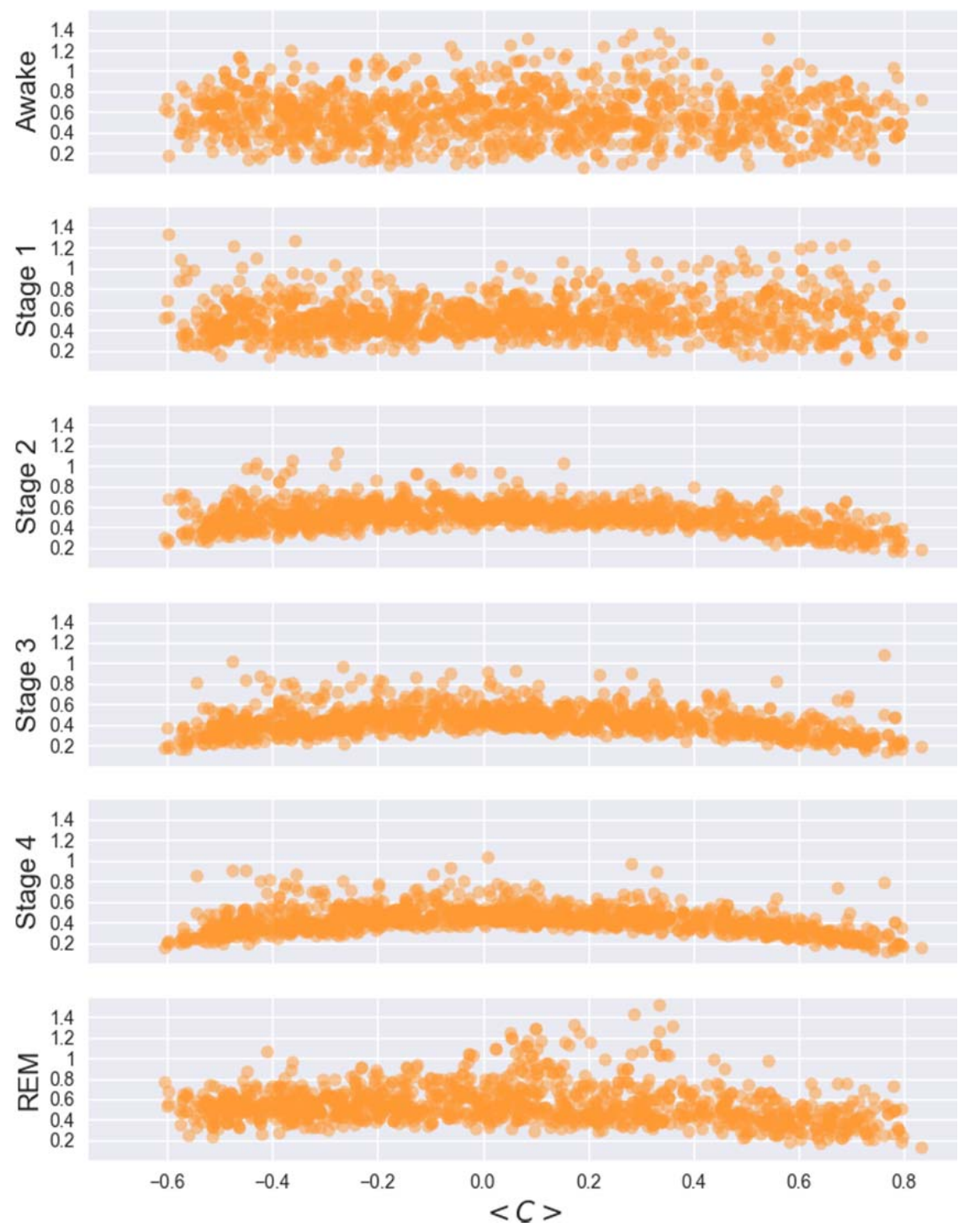

Figure 6: Size of the $90 \%$ confidence interval of cross-correlation coefficients in each sleep stage (ordinate) versus their mean value (abscissa) for all 10 sleep recordings. 
1 Supplementary Material of "Characteristic Fluctuations around Stable Attractor Dynamics extracted from highly non-stationary Electroencephalographic Recordings".

Paola V. Olguín-Rodríguez ${ }^{1, a}$, J. Daniel Arzate-Mena ${ }^{1, b}$, Maria Corsi-Cabrera ${ }^{2,3, c}$, Heidemarie Gast $^{4, d}$, Arlex Marín-García ${ }^{5, e}$ Johannes Mathis ${ }^{6, f}$, Julieta Ramos Loyo ${ }^{7, g}$, Irma Yolanda del Rio-Portilla ${ }^{2, \mathrm{~h}}$, Christian Rummel ${ }^{6, \mathrm{i}}$, Kaspar Schindler $^{4,6, \mathrm{j}}$, Markus Müller ${ }^{8,9, \mathrm{k}}$

${ }^{1}$ Instituto de Investigación en Ciencias Básicas y Aplicadas, Universidad Autónoma del Estado de Morelos (UAEM), Cuernavaca Morelos, México.

${ }^{2}$ Facultad de Psicología, Universidad Nacional Autónoma de México (UNAM), Distrito Federal, México.

${ }^{3}$ Unidad de Neurodesarrollo, Instituto de Neurobiología, Universidad Nacional Autónoma de México (UNAM), Juriquilla, Querétaro, México

${ }^{4}$ Department of Neurology, Inselspital, Bern University Hospital, University Bern, Bern, Switzerland.

${ }^{5}$ Instituto de Ciencias Físicas (ICF), Universidad Nacional Autónoma de México (UNAM), Cuernavaca Morelos, México

${ }^{6}$ Support Center for Advanced Neuroimaging, University Institute for Diagnostic and Interventional Neuroradiology, Inselspital, Bern University Hospital, University Bern, Bern, Switzerland.

${ }^{7}$ Instituto de Neurociencias, Universidad de Guadalajara.

${ }^{8}$ Centro de Investigación en Ciencias, Universidad Autónoma del Estado de Morelos (UAEM), Cuernavaca Morelos, México.

${ }^{9}$ Centro Internacional de Ciencias A. C., Cuernavaca, Morelos, México

ㅁane23.star@gmail.com, ${ }^{b}$ jd.arzate@gmail.com, ${ }^{c}$ corsi@unam.mx, dheidemarie.gast@gmail.com, earlex.marin@gmail.com, 
Page 53 of 69

fiohannes.mathis@belponline.ch, ${ }^{,}$iramos@cencar.udg.mx, ${ }^{\text {hiyrp@unam.mx }}$

'crummel@web.de, ${ }^{j}$ kaspar.schindler@gmail.com, ${ }^{k}$ muellerm@uaem.mx

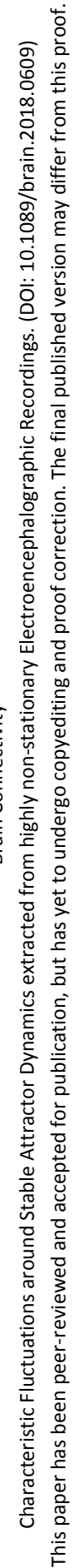




\section{Average Matrices of resting state EEGs:}

Like Figure 1 in the main text we provide here mean correlation matrices of resting state data averaged separately for eyes open and eyes closed condition. Shown is the correlation pattern for two subjects, one belongs to the group of young the other to the group of elderly adults. The similarity of the correlation pattern is intriguing. A similar figure for an average correlation matrix for the pre-seizure, seizure and post-seizure period of a focal onset seizure can be found in (Müller et al. 2014) . 

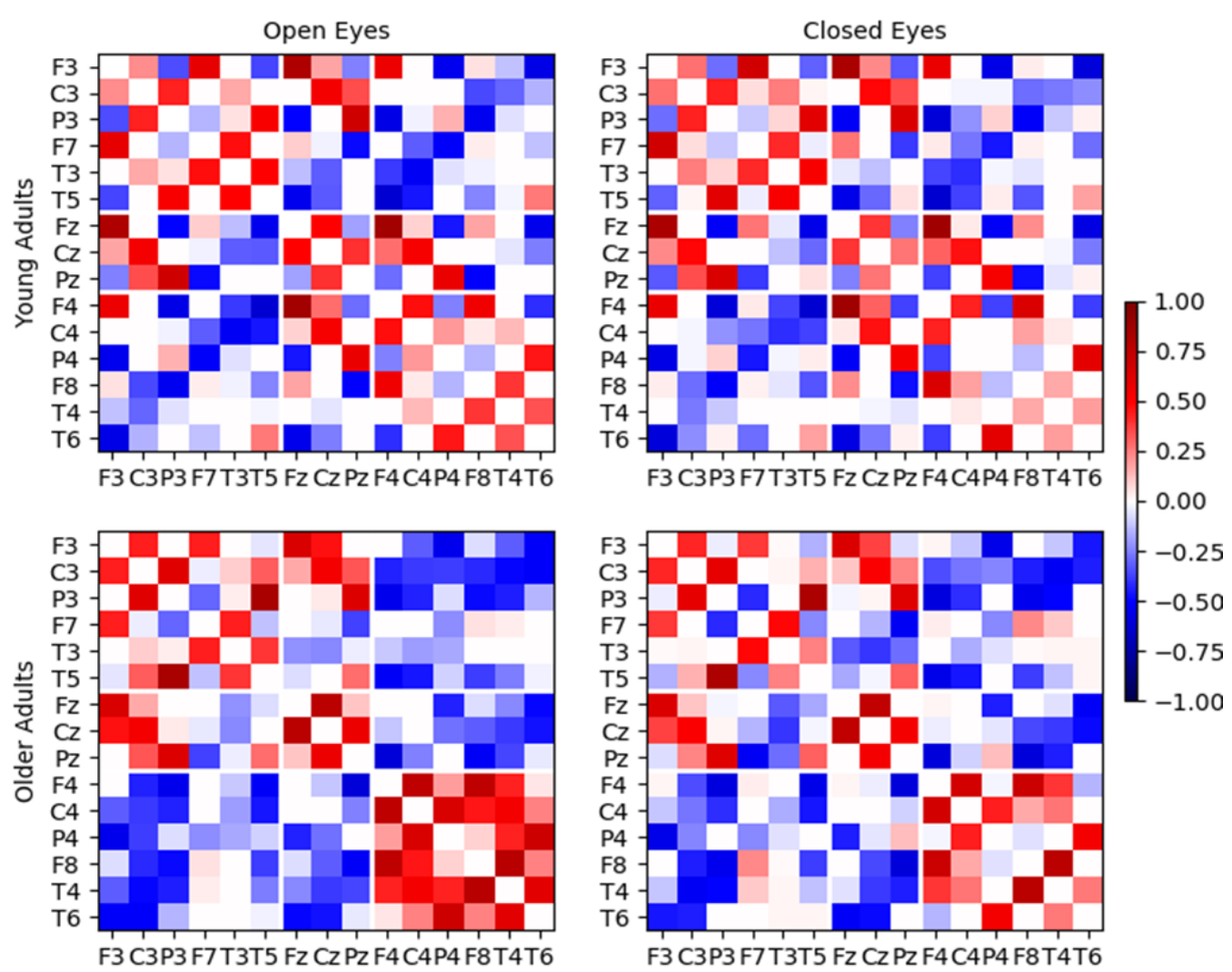

Figure S1 Average correlation matrices obtained from resting state EEGs for eyes open and eyes closed condition of a young and elderly adult. 
Comparison of average matrices obtained separately for physiological states:

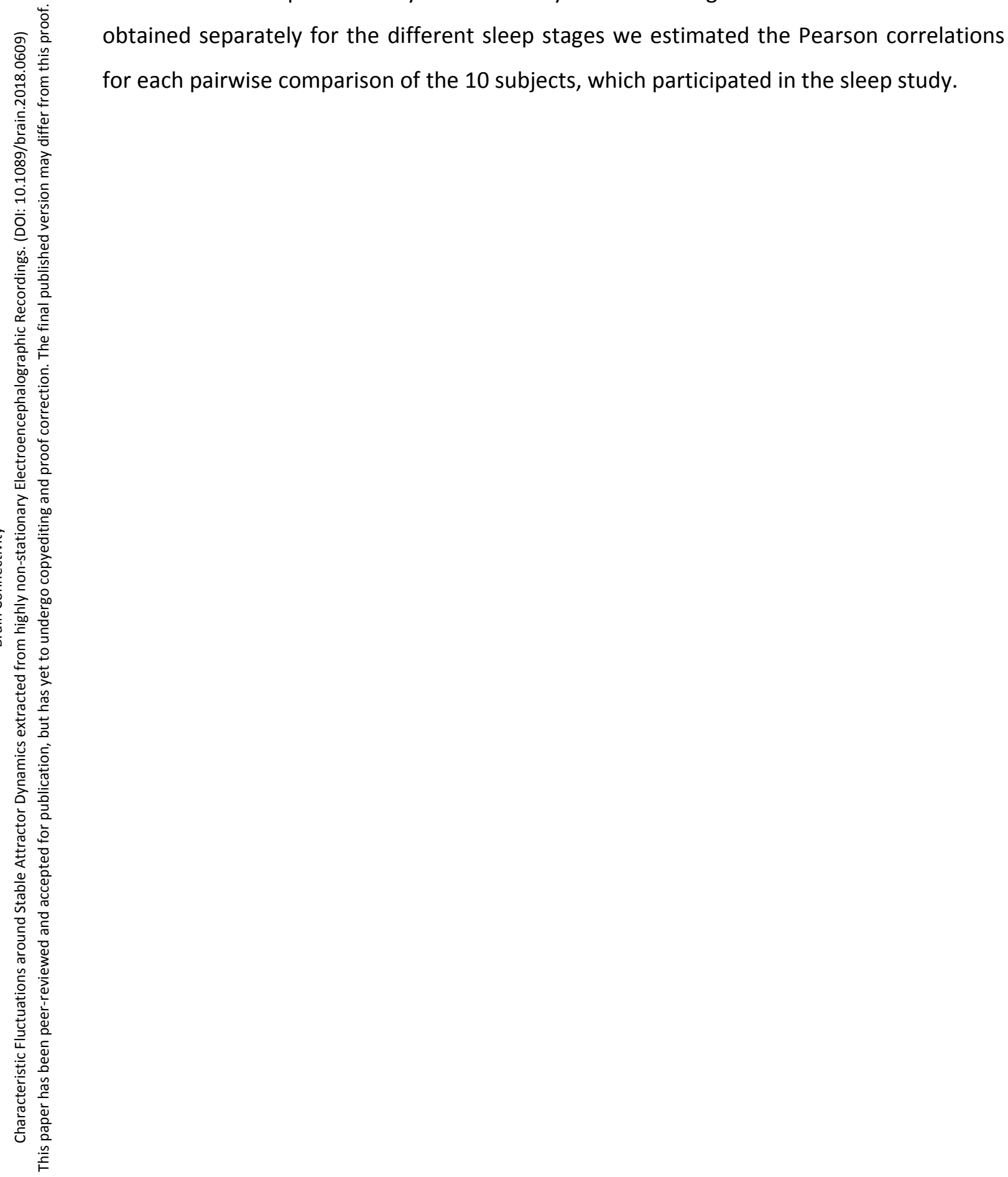




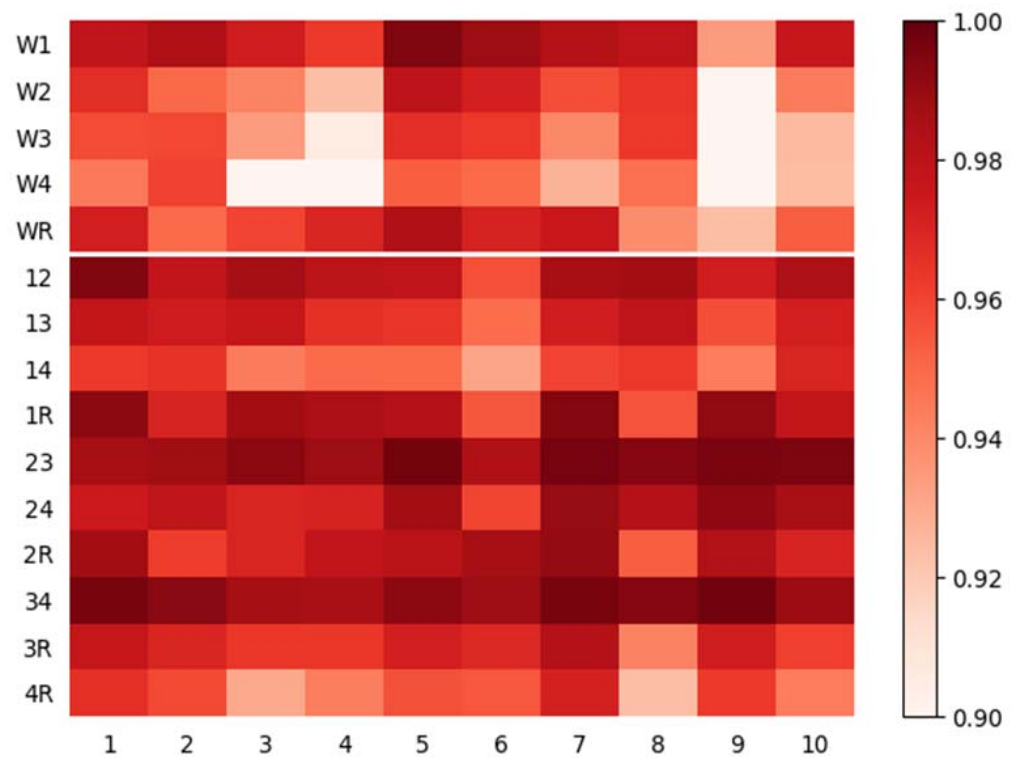

Figure S2: Pearson Coefficients between all pairs of the average correlation matrices obtained separately for the different sleep stages of each subject. The shortcuts at the ordinate denote: $\mathrm{R}=\mathrm{REM} ; \mathrm{W}=$ waking and $1,2,3,4$, the non-REM sleep stages. The abscissa enumerates the subjects. Note, the color scale initiates only at 0.9. 
At first glance, all correlation coefficients shown in Figure $\mathrm{S} 2$ are notably high, namely, they are larger than 0.9 , which testifies the extraordinary similarity of the average matrices obtained for different sleep stages. Second, the waking state shows lowest similarity to all sleep stages, as could be anticipated intuitively. Apparently, structural differences of the average correlations are more pronounced between the sleeping and the non-sleeping brain than between any pair of sleep stages. Furthermore, one observes in Figure S2 that the average correlation pattern obtained for NREM sleep stages 2, 3 and 4, as well as that for 1 and 2 are also quite similar. The highest structural discrepancies between sleep stages can be observed for the comparison of NREM sleep stage 4 and REM as well as NREM 1 and 4. The correlation between the average correlation matrices between sleep stages 1 and 2 is somewhat lower, but still documents a high structural similarity. The same is true for the waking state and sleep stage 1.

The present results are consistent with the two mechanisms involved in the generation of EEG signals. One is the thalamus-cortical oscillator and the other one the brain-stem activating system (an important bottom-up activating system), which is responsible for alertness. Cortical EEG sleep oscillations depend on the firing mode of the Thalamuscortical neurons. During NREM sleep the firing mode changes from the tonic mode, characteristic of activated states that are particularly active during the waking state and REM sleep, to the oscillatory mode of NREM sleep, when thalamus-cortical neurons become hyper-polarized while generating Delta waves and sleep spindles depending on the level of hyper polarization (Steriade 1997; Steriade and McCarley, 1990). The presence of such oscillations has been shown in an animal model, where Delta-waves as well as sleep spindles have been found zero-lag correlated between different cortical locations (Contreras et al., 1997; Destexhe et al., 1999). Steriade and coworkers found such synchronized oscillations with intra-cranial electrodes placed upon the cortex. Here zerolag correlations can not be explained via volume conduction effects. Such phenomenon has also been observed in humans by analyzing extra-cranial recordings (Guervara et al., 1995; Achermann and Borbely, 1998). The two mechanisms, one active in waking stage and REM Sleep and, the other respectively, during NREM-sleep, mark qualitative differences between stage 4 and REM as well as between stages 1 and 4 respectively. 
From this perspective the largest discrepancy should be expected between NREM sleep and waking state, and also between $\mathrm{W}$ and REM sleep, whereas, stages 2, 3 and 4 of NREM sleep are characterized by increasing amount of Delta activity according with both the standard manual (Rechtschafen and Kales, 1968,Lesser et al.,1986). Accordingly, it could be expected that the highest correlation is observed between stages 2 and 3 as well as between stages 3 and 4 . Stage 1, on the other hand, is a transitional state between $\mathrm{W}$ and sleep with mixed EEG activity, when alertness is already reduced but sleep promoting mechanisms of the thalamus-cortical oscillatory mode are still not fully installed (CorsiCabrera et al., 2006). That may explain the closeness between $W$ and stage 1 ; another possible explanation is that, although during stage 2 the oscillatory mode is already working, the transition from stage 1 to stage 2 is based on the presence of at least two sleep spindles and/or K-complex and a low amount of Delta, introducing possible arbitrary difference of classification. A third explanation the short duration and the small overall amount of sleep stage 1 periods during night.

In conclusion, these findings provide a quantitative justification for averaging over the whole EEG-recordings, without any distinction between sleep stages.

Analogously to the results presented in Figure S2 we also estimated the Pearson coefficient for the comparison of the eyes open and closed condition of the resting state EEGs. 


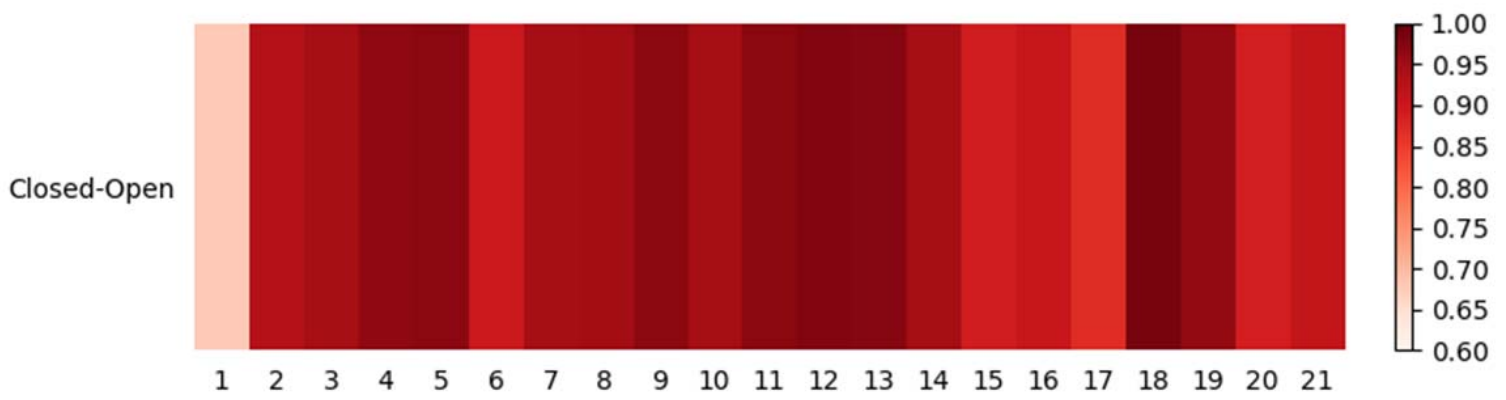

$\begin{array}{lllllllllllllllllllll}1 & 2 & 3 & 4 & 5 & 6 & 7 & 8 & 9 & 10 & 11 & 12 & 13 & 14 & 15 & 16 & 17 & 18 & 19 & 20 & 21\end{array}$

Figure S3: Pearson Coefficients for the comparison of average correlation matrices obtained separately for the eyes closed and eyes open condition of the resting state EEGs. With the exception of recording 1 all estimates are notably above 0.8

Like in Figure S2 and Figure 2 of (Müller et al. 2014) the similarity of the average spatial correlation structure for the two resting state conditions is extremely high. These results justify the average over both conditions. The resulting matrix is called the stationary pattern of the resting state EEGs. 
Comparison of the overall correlation strength by using the Mann-Whitney-Wilcoxon rank test:

A)

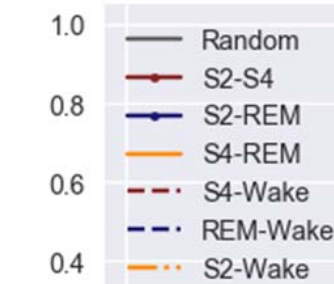

B)
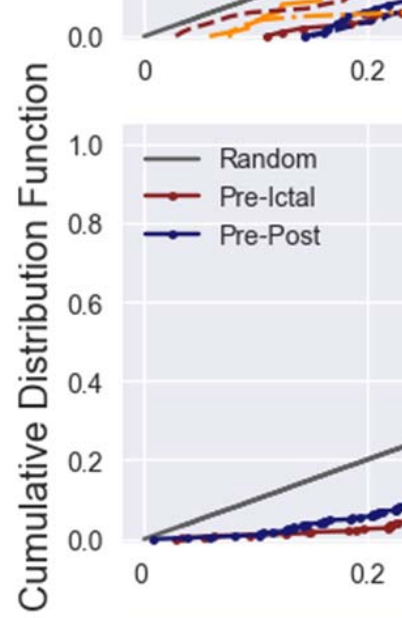

0.4

0.6

1

C)

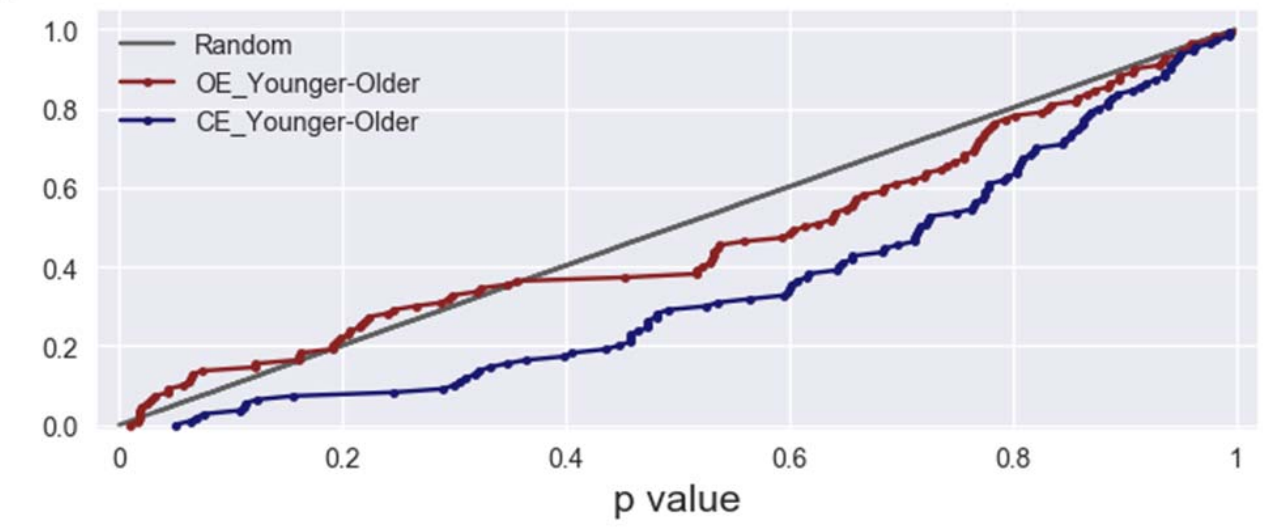

Figure S4: Pairwise comparison cross-correlation matrices averages separately for different physiological states. P-value are estimated by the non-parametric Mann-WhitneyWilcoxon-rank test. Panel A provides the pairwise comparison of different sleep stages, panel B comparison of average correlation matrices derived from the pre-seizure, seizure 

and post-seizure period, C comparison of the results derived from Young and elderly adults with open and closed eye condition.

Figure S4 provides a detailed comparison of average correlation matrices derived from different physiological stages by employing the Mann-Whitney-Wilcoxon rank test. None of the comparisons lead to significant results, because the average spatial correlation pattern remains extremely similar in all cases considered in this study. 


\section{Attractor Dynamics, transient behavior and non-stationarity}

In the main text the stable scaffold of spatial interrelations has been interpreted as a shadow of the dynamics on (or close to) the attractor in phase space and non-stationary dynamical features has been linked to specific excursions from the invariant set. This led to the assumption that different physiological states are expressed by characteristic deviations from the stationary pattern, which could be confirmed for the peri-ictal transition of epileptic seizures, the different sleep stages and the eyes open/eyes closed condition during rest. It turned out that average difference matrices are not zero but show characteristic deviations if estimated for different physiological states separately. Furthermore, this picture drawn from dynamical system theory fits nicely to the abundant empirical evidence suggesting that the brain is a complex system operating close to the critical point of a second order phase transition and is also congruent with the lavish energy consumption of the brain even at resting state.

Nonetheless, this is a hypothetical statement, given that an adequate reconstruction of the true phase space of the brain activity, and though a direct proof that the attractor of the brain dynamics is directly responsible for the observed stable correlation pattern is principally impossible due to its astronomically high dimension. However, at least the opposite direction, namely, that a frequently perturbed and, hence, non-stationary dynamical system provides the same correlation pattern as the unperturbed one, while qualitative changes of the attractor lead to qualitative changes of cross correlations can be shown easily.

To this end we performed to types of calculation. In a first step we considered two diffusively coupled non-identical Rössler systems (cite Rosenblum):

$$
\begin{gathered}
x_{1} \cdot(t)=-\omega_{1} y_{1}(t)-z_{1}(t)+\eta\left(x_{2}(t)-x_{1}(t)\right) \\
y_{1} \cdot(t)=\omega_{1} x_{1}(t)-\beta y_{1}(t) \\
z_{1} \cdot(t)=0.2+z_{1}(t)\left(x_{1}(t)-10\right) \\
x_{2} \cdot(t)=-\omega_{2} y_{2}(t)-z_{2}(t)+\eta\left(x_{1}(t)-x_{2}(t)\right)
\end{gathered}
$$




$$
y_{2} \cdot(t)=\omega_{2} x_{2}(t)-\beta y_{2}(t)
$$

$z_{2} \cdot(t)=0.2+z_{2}(t)\left(x_{2}(t)-10\right)$

$$
\text { where } \omega_{1} \text { and } \omega_{2} \text { are } 0.985 \text { and } 1.05 \text { respectively, } \beta=0.15 \text { and } \eta=0.2 \text {. }
$$

This system was then integrated with a $4^{\text {th }}$ order Runge Kutta method using a step with of 0.05. From the resulting 6 dimensional multivariate recording we then estimated the cross correlation matrix over $2^{16}$ data points.

The attractor of the first Rössler system and the results for the estimation of the average cross-correlation matrix are displayed in Figure S5.

For comparison we then repeated the same computation for the corresponding perturbed system. To this end we added independent Gaussian random numbers (zero mean, standard deviation equal to 2 ) to each of the $x$ and $y$ components at every 250 time steps. The same is done for both $z$ components, but now absolute values have been taken in order to avoid negative values. Furthermore, we reset $z$ components below 10 if they increase above 25 in order to prevent divergence. Note, this system is non-stationary by definition, given that it moves almost always on a transient around the attractor. As displayed in Figure S5, the corresponding trajectory in phase space is notably distorted in comparison to Figure S5. However, the mean correlation pattern is not affected by such a drastic intervention. 
A)

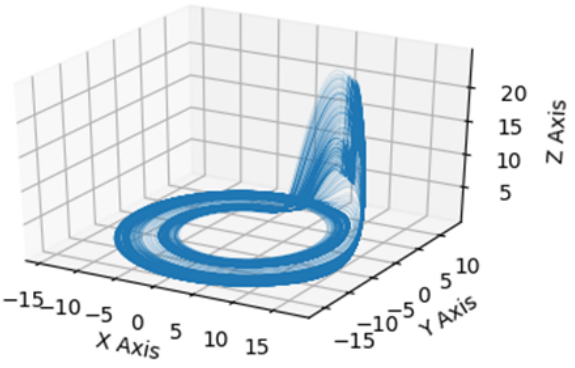

B)

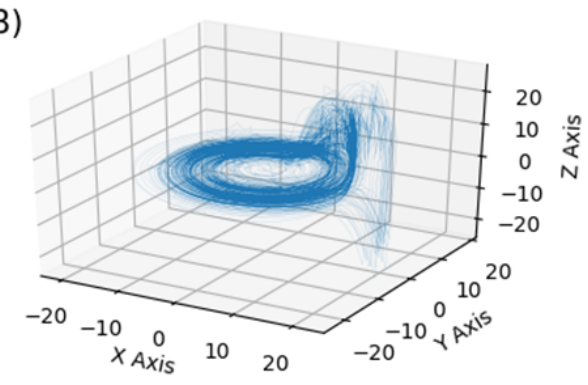

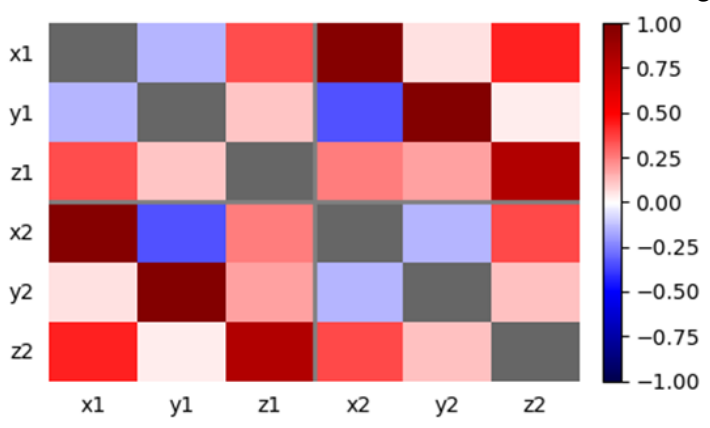

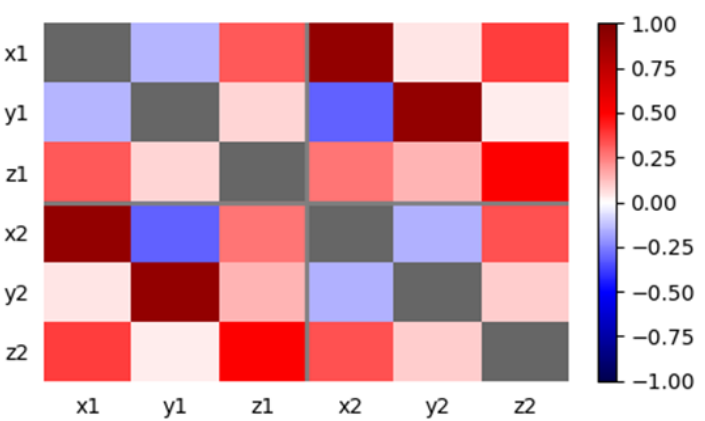

Figure S5: A) Attractor of one of the coupled Rössler system and B) the corresponding correlation matrix of the complete system. C) and D) shows the same for the strongly perturbed system. 
In a second step we created non-stationary signals by considering a mixed Rössler-Lorenz system. Here we used the equations S1-S6 and a anti-correlated Lorenz-system:

$$
\begin{gathered}
x_{1} \cdot(t)=\sigma\left(y_{1}(t)-x_{1}(t)\right)+0.3\left(x_{1}(t)-x_{2}(t)\right) \\
y_{1} \cdot(t)=R x_{1}(t)-y_{1}(t)-\left(x_{1}(t) z_{1}(t)\right) \\
z_{1} \cdot(t)=-b_{1} z_{1} \frac{(t)}{b_{2}}+y_{1}(t) x_{1}(t) \\
x_{2} \cdot(t)=\sigma\left(y_{2}(t)-x_{2}(t)\right)+0.3\left(x_{2}(t)-x_{1}(t)\right) \\
y_{2} \cdot(t)=R x_{2}(t)-y_{2}(t)-\left(x_{2}(t) z_{2}(t)\right)
\end{gathered}
$$

$z_{2} \cdot(t)=-b_{2} z_{2} \frac{(t)}{b_{2}}+y_{2}(t) x_{2}(t)$

with $\sigma=10, b_{1}=8, b_{2}=3$ and $R=28$.

Time series are then created by a mixed state $\Theta_{i}(t)=(1-\rho) \Theta_{i}^{R}(t)+\rho \Theta_{i}^{L}(t)$

respectively, while $\rho$ increases gradually from zero to one. In total we derived $2^{17}$ data points, such that in the first part the Rössler systems dominate while it is governed by the Lorenz systems in the second half. The phase space trajectory of such a mixed system for the first and second half as well as the corresponding correlation matrices are shown in Figure S6. 
A)

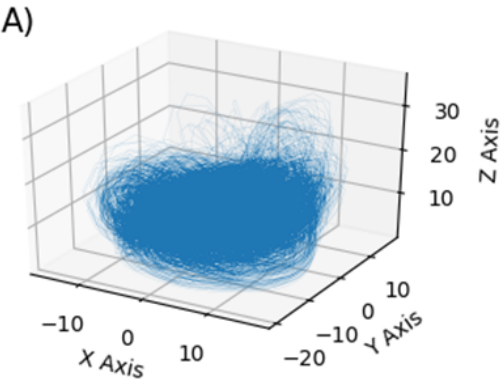

B)

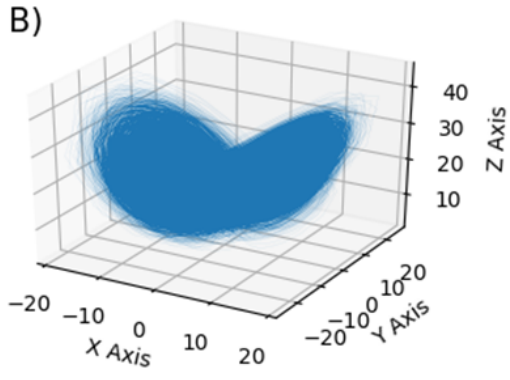

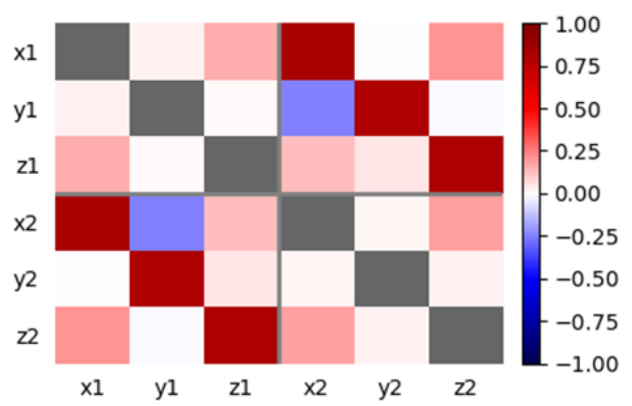

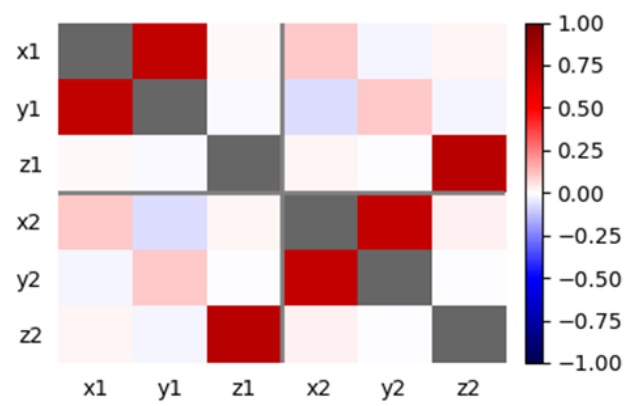

Figure S6: Results obtained for the mixed Rössler-Lorentz system. A) shows the mixed attractor of the derived from the first half of the time series, panel B) the same for the second half of the time series. Panel B) and D) show the corresponding correlation matrices derived from the complete 6 dimensional system.

One observes nicely that the geometry of the phase space trajectories change qualitatively, which results in qualitatively different mean cross-correlation matrices. Evidently, the situation shown in Figure S5 is much more affine to the observations reported in the main text. In both cases we are considering highly non-stationary systems, but the first one leads to stationary cross-correlations while the mean cross-correlation matrix is time dependent in the second case.

If one destroys, on the other hand, any deterministic feature imprinted in the signals, no mutual correlations remain and estimates of the cross-correlation matrix are close to unity in matrix space.

The complete information about the geometry in phase space is imprinted in (measured or numerically estimated) time series, a fact that allows the reconstruction of topologically equivalent phase space structures via e.g. embedding procedures. Parseval's theorem, on the other hand, ensures that the amount of information imprinted in a time series is 
68 identical to the information content of its Fourier Transform. Given that the linear autocorrelation function is equivalent to the power spectrum, the Fourier Amplitudes do not contain any information about the attractor topology. The complete information about the deterministic structure of an dynamic process is solely induced in the Fourier Phases. Thus phase randomized signals carry the same linear univariate properties as the original time series but are of stochastic nature otherwise. This can be achieved by Iterative Amplitude Adjusted Fourier Transform Surrogates (IAAFT) (Kanz-Schreiber), where Fourier Phases get randomized, while Power Spectra as well as Amplitude Distributions are preserved. However, such procedure also destroys linear interrelations between time series and though cross-correlation estimates are close to zero.

We acknowledge that the above considerations do not constitute a strict proof for the correctness of our interpretation, but, together with the above mentioned evidence, it makes it at least more plausible and substantiates the consistent explanation of the observed phenomena in terms of dynamical system theory provided in the main text. 


\section{References:}

Achermann P, Borbély AA (1998) Coherence analysis of the human sleep electroencephalogram. Neuroscience 85:1195-120

Contreras, D., Destexhe A., Terrence, J., Sejnowski, T.J., Steriade, M. Spatio temporal patterns of spindle oscillations in cortex and thalamus. J Neurosci. 1997; 17 (3): 1179-1196.

Corsi-Cabrera, M., Del-Río-Portilla, Y., Muñoz-Torres, Z. Power and coherent oscillations distinguish REM sleep, stage 1 and wakefulness. International Journal of Psychophysiology, 2006, 1006; 60: 59-66

Destexhe, A., Contreras, D., Steriade, M. Spatiotempral analysis of local field potentials and unit discharges in cat cerebral cortex during natural wake and sleep states. J Neurosci., 1999; 19 (11): 4595-4608.

Guevara, M.A., Lorenzo, I., Arce, C., Ramos, J., Corsi-Cabrera, M., (1995). Inter- and intrahemispheric EEG correlation during sleep and wakefulness. Sleep 18, 257-265.

Lesser, R. P. (1986). Guideline seven: A proposal for standard montages to be used in clinical EEG. Journal of Clinical Neurophysiology, 3(3 SUPPL. 1), 26-33.

Müller MF, Rummel C, Goodfellow M, Schindler K (2014). Standing Waves as an Explanation for Generic Stationary Correlation Patterns in Noninvasive EEG of Focal Onset Seizures, Brain Connectivity, 4: 131-144

Rechtschaffen, A., Kales, A. (Eds.), 1968. A Manual of Standardized Terminology, Techniques and Scoring System for Sleep Stages of Human Subjects. Brain Information Service, Brain Research Institute, University of California, Los Angeles.

Steriade,M., 1997. Synchronized activities of coupled oscillators in the cerebral cortex and thalamus at different levels of vigilance. Cereb. Cortex 7, 583-604.

Steriade M, McCarley RW. Brainstem control of wakefulness and sleep. New York: Plenum Press, 1990. 